\title{
UNA CLASIFICACIÓN FUNCIONAL DE LAS PRINCIPALES CIUDADES DE MÉXICO
}

\author{
LUIS UNIKel y Gustavo Garza * \\ El Colegio de México
}

\section{INTRODUCCIÓN}

Clasificar ciudades no es un ejercicio novedoso. ${ }^{1}$ No obstante, se manifiesta un interés persistente por clasificarlas con el propósito de describir o explicar algunas de sus características.

Los primeros trabajos sobre el tema han identificado ciudades industriales, comerciales, de servicios, turísticas, mineras y otras clases funcionales, atendiendo a su estructura ocupacional con respecto a la de otras ciudades aceptadas a priori como típicas en cada una de las categorías mencionadas. A medida que se han refinado los métodos analíticos aplicados para clasificarlas, el número de tipologías de ciudades se ha multiplicado. Teóricamente no hay límite al número de clasificaciones, dada la diversidad de criterios metodológicos, de las variables que se utilizan y de la unidad territorial que se seleccione.

A pesar del gran esfuerzo desplegado en esa creciente serie de estudios, persiste la polémica sobre la validez de dichos intentos y por ello los analistas de las ciudades continúan determinando nuevas clasificaciones con la intención de superar a las establecidas o para satisfacer mejor sus objetivos. En conclusión, se puede decir que no hay clasificaciones correctas o falsas, sino más bien útiles, en mayor o menor grado, para fines específicos.

En el presente trabajo se pretende determinar una clasificación de las principales ciudades de México (50000 y más habitantes en 1960) según su función predominante, utilizando como característica fundamental la estructura de la mano de obra residente por ramas de acti-... vidad. Esta tipología, la más ampliamente utilizada, fue seleccionada tanto por consideraciones teóricas como por ajustarse a la información estadística disponible.

Desde un punto de vista teórico algunos autores expresan razones de peso para aplicar una clasificación funcional o de actividad econó-

* Agradecemos a Omar Lazcano su colaboración en la revisión final de este trabajo.

1 Las primeras y más obvias clasificaciones de ciudades se han hecho con base en el tamaño de su población. De acuerdo con otras características, particularmente la fuerza de trabajo, las clasificaciones se han incrementado sustancialmente a partir de los años cincuenta. Para conocimiento y comentarios sobre las principales clasificaciones funcionales de ciudades, véase: J. Halden y $\mathrm{E}$. Borgatta, American Cities, Chicago, Rand McNally, 1965, y R. Murphy, The American City, Nueva York, 1966, pp. 113-129. 
mica predominante. ${ }^{2}$ La principal de ellas es el reconocimiento de la interdependencia de los sectores dominantes de la organización económica de un complejo urbano con otras características de su población. Pero ésta, como otras aseveraciones sobre el tema, es una hipótesis más que una teoría probada empíricamente. Precisamente, los autores del presente trabajo reconocen la escasa base teórica que fundamenta una clasificación funcional económica y, por lo mismo, la relativa arbitrariedad implícita en el método aplicado en este estudio, pero que, de hecho, se presenta en todo ensayo taxonómico.

La clasificación de ciudades determinada no pretende ser un fin en sí mismo, ni ser (metodológicamente hablando) una más de las muchas existentes. Se considera como uno de los primeros intentos de aplicar sistemáticamente en México un instrumento analítico para explorar las relaciones entre la especialización económica y algunas características básicas de la población urbana. ${ }^{3}$ El propósito último es aportar un elemento adicional a la descripción del proceso de urbanización en México.4

Con la clasificación obtenida se pretende contestar las siguientes interrogantes: a) ¿cuáles y cuántos tipos de ciudades (de las 37 estucliadas) se presentan y cuál ha sido su evolución de 1940 a 1960? b) ¿cuál ha sido la probable tendencia de esta evolución de 1960 a 1970 ? c) ¿qué asociación existe entre las distintas funciones económicas predominantes de las ciudades con el tamaño y la tasa de crecimiento de su población, así como con la ubicación geográfica de las mismas? y d) ¿qué tipo de ciudades contienen las distintas zonas en que se ha dividido al país según el nivel de desarrollo económico? 5

Se desea que este trabajo, cuyo tema de investigación es nuevo en México, no obstante sus limitaciones, estimule a otros estudiosos de la urbanización a profundizar en la materia. También se espera que sirva, en la práctica, como uno de tantos elementos a tomar en cuenta en las tareas que se realizan para el planeamiento del desarrollo urbano y regional del país.

\section{FUNCTONES ECONÓMICAS PREDOMINANTES}

No obstante que la ciudad juega un papel crucial en el desarrollo nacional y regional, los planificadores de la economía conceden poca

$2 \mathrm{~T}$. Wilkinson, The Urbanization of Japanese Labor, 1868-1955, The University of Massachusetts Press, 1965; H. Mayer y C. Kohn, (Eds.), Readings in Urban Geography, Chicago, The University of Chicago Press, 1960, pp. 127-128.

3 Una de las pocas clasificaciones conocidas para México es la elaborada por R. Cinta, quien agrupó 100 municipios según cinco tipos de ramas de actividad principal en 1960. La clasificación se hizo en función del porciento de población económicamente activa de la total de cada municipio. Véase R. Cinta, Aspectos socioeconómicos de la urbanización en México, tesis, Escuela Nacional de Ciencias Políticas y Sociales, UNAM, México, 1967.

4 Este estudio corresponde al proyecto 2 del programa de investigación sobre el proceso de urbanización en México. Véase L. Unikel et al., Ei proceso de urbanización en México: anteproyecto de investigación, Centro de Estudios Económicos y Demográficos, EI Colegio de México, 1967.

5 La regionalización aplicada corresponde a 1960 y se obtuvo a partir de la relación producto bruto por hombre ocupado. Véase L. Unikel, G. Garza y A. Gon- 
atención a la urbanización y a la importancia de organizar las funciones y actividades que se conjugan en el contexto urbano. ${ }^{6}$ México no es una excepción a esta observación.

La elevada tasa de incremento de la población total del país (3.4\% anual de 1960 a 1970) aunada a la intensa migración campociudad ha dado por resultado el crecimiento rápido de las ciudades existentes y la multiplicación de nuevos centros urbanos. ${ }^{7}$ Simultáneamente se ha dado una concentración del producto en las principales ciudades y, en particular, en la zona metropolitana de la ciudad de México. Por ejemplo, los municipios que contienen las 37 áreas urbanas estudiadas generaron, en 1965, el $90.2 \%$ del valor de la producción industrial (transformación, construcción, electricidad y gas), el $74.7 \%$ de los ingresos brutos por servicios y el $39.3 \%$ del valor de las ventas de mercancías realizadas en México. La zona metropolitana de la ciudad de México absorbió, en esas fechas, $42.8 \%, 49.5 \%$ y $15.2 \%$, respectivamente. 8

Las ciudades presentan diferencias significativas, tanto en su estructura productiva como en su población económicamente activa. En términos generales, se sabe del predominio de ciertas actividades económicas en algunas ciudades del país. Tal es el caso de Monterrey y Orizaba, ciudades industriales; de la ciudad de México, que concentra toda clase de actividades; el puerto de Veracruz, especializado en transportes; Tijuana y Acapulco en servicios, etc. Sin embargo, esta tipología sólo se puede utilizar con alguna confianza para el caso de ciudades que tradicionalmente se han especializado en una sola actividad. Su fundamentación es intuitiva y no producto de un análisis empirico.

La clasificación que se presenta abarca a las 37 mayores áreas urbanas del país: las que contaban con 50000 y más habitantes en 1960. Para el efecto se aplicó el "índice de trabajadores excedentes" 9 a la información censal ajustada de la población económicamente activa de 1940,1950 y 1960 , de tal modo que permitiera su análisis comparativo en el tiempo.10

zález, "Urbanización y desarrollo económico en México", ponencia presentada a la Conferencia Rohovot sobre urbanización y desarrollo en países subdesarrollados, Rohovot, Israel, agosto de 1971.

6 M. Rivkin, "Urbanization and National Development", en Naciones Unidas, Urbanization: Development Policies and Planning, Nueva York, 1968, p. 79.

7 Para mayores detalles, véase L. Unikel, "Urbanización", en Dinámica de la población de México, Centro de Estudios Económicos y Demográficos, El Colegio de México, 1970, pp. 115-147.

8 Cálculos elaborados con datos de los censos económicos (no publicados) de la Dirección General de Estadística.

9 La expresión matemática del índice es la siguiente: $I_{t e}=\frac{e_{i}}{e_{t}}-\frac{E_{i}}{E_{+}}$, en donde $e_{i}$ y $E_{i}$ son la PEA total, local y nacional en la rama de actividad $i$, y $e_{t}$ y $E_{t}$ son la PEA total, local y nacional, respectivamente. Tal como el nombre del índice lo expresa, el excedente absoluto y relativo de trabajadores de una ciudad con respecto al total nacional (o algún otro denominador totalizador) se considera una expresión de predominio o especialización en dicha actividad. Para mayores detalles véase el apéndice.

10 Para mayores detalles sobre el ajuste de la información censal de la PEA y del nivel municipal al de ciudad, véase $L$. Unikel y $F$. Torres, "La población 


\section{Cuadro 1}

MÉXICo: Ciudades SegúN NÚmero de habitantes, funciones PRedominantes Y UBICACIÓN GEOGRÁFICA, 1940-1960

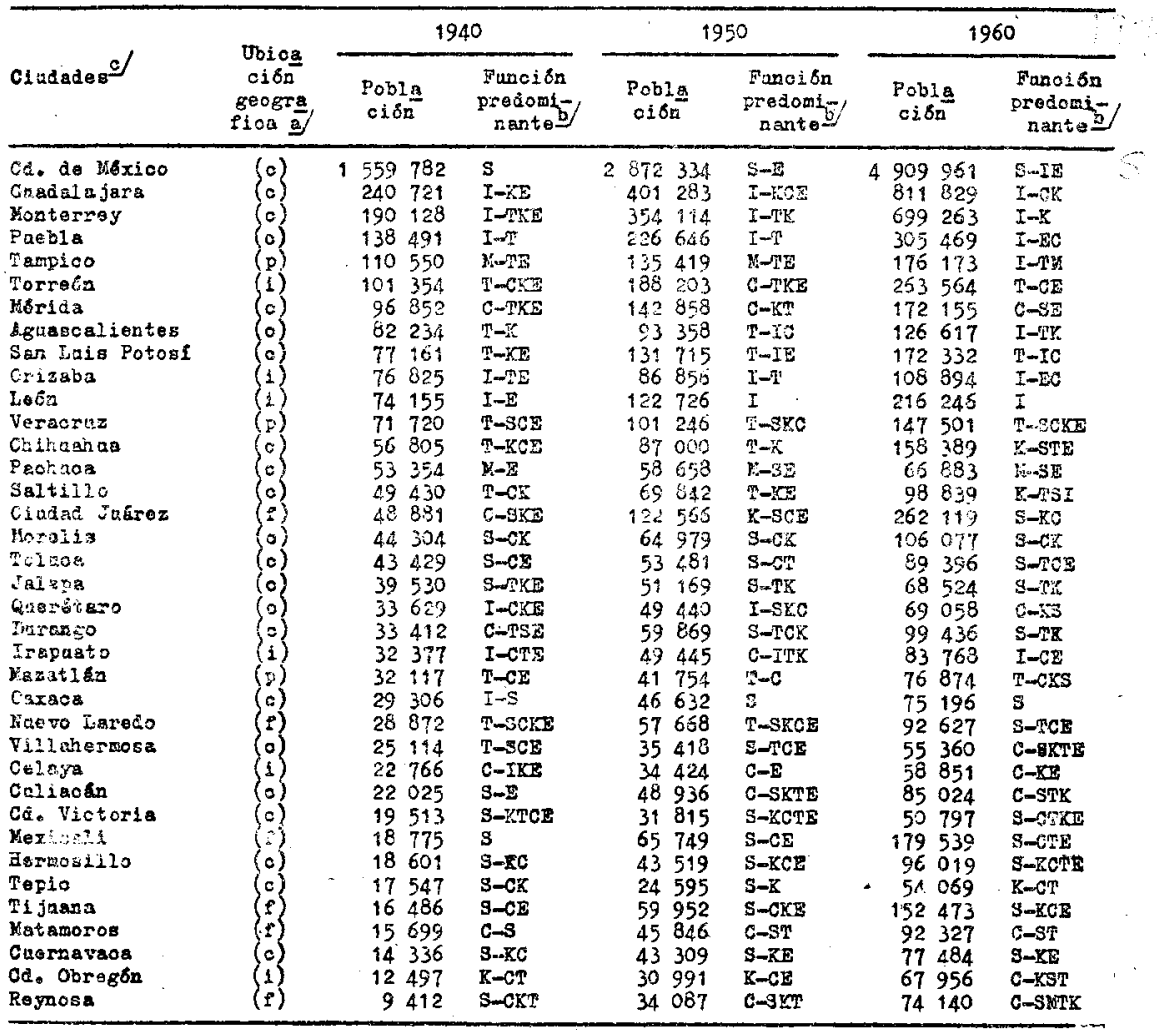

Fuentes: La población de las ciudades se obtuvo de L. Unikel, "El proceso de urbanización en México", Demografía y Economía, Vol. II, Núm. 2, El Colegio de México, 1968, cuadro 20. La función predominante fue calculada según la fórmula presentada en el apéndice y con base en los datos de los cuadros A1, A2 y A3.

a (c) ciudades capitales; (f) fronterizas; (i) del interior; (p) portuarias.

b S, servicios; I, industria de transformación; $\mathrm{C}$, comercio; $\mathrm{T}$, comunicaciones y transportes; $K$, construcción; $E$, electricidad y gas; $M$, industria extractiva.

c Los datos corresponden, por lo menos en uno de los tres años estudiados, a las áreas urbanas de estas ciudades - la ciudad central más la periferia de contiguiidad física-, excepto en once de ellas. Véase Unikel, "El proceso de urbanización...", loc. cit., cuadro 20.

El número y tipo de actividades económicas predominantes según la población económicamente activa (PEA) se presentan en el cuadro 1.11 Para su mejor entendimiento se ilustra el caso del área urbana de Monterrey en 1940, cuya clasificación se la ha designado como

económicamente activa en México y sus principales ciudades, 1940-1960", DEMoGRAFÍa y Economía, Vol. IV, Núm. 1, El Colegio de México, 1970, pp. 38-42.

11 Sería sumamente interesante obtener una clasificación similar según el 
$I-T-K-E$. Esto significa que: a) la actividad económica predominante es la industria de transformación $(I) ; b$ ) que le siguen en importancia decreciente, comunicaciones y transportes $(T)$; construcción $(K) \mathrm{y}$, finalmente, electricidad y gas $(E)$.

Número de funciones predominantes. Atendiendo a las diferencias que se presentan en el número de funciones predominantes (cuadro 1), los centros urbanos se clasificaron en cuatro tipos: unifuncionales, bifuncionales, semidiversificados y diversificados, según resultaron con una, dos, tres y cuatro o más funciones predominantes, respectivamente.

\section{Cuadro 2}

México: Número y Porciento de ciUdades SegúN el Número de actividades ECONOMICAS PREDOMINANTES, 1940-1960.

\begin{tabular}{|c|c|c|c|c|c|c|}
\hline \multirow{2}{*}{$\begin{array}{l}\text { Civiades segun nú } \\
\text { mero de funciones } \\
\text { predominantes }\end{array}$} & \multicolumn{2}{|c|}{1940} & \multicolumn{2}{|c|}{1950} & \multicolumn{2}{|c|}{1960} \\
\hline & Núre ro & $\%$ & Núme ro & $\%$ & Níme ro & $\%$ \\
\hline Total & 37 & 100.0 & 37 & 100.0 & $3 ?$ & 100.0 \\
\hline $\begin{array}{l}\text { Unifuncionales } \\
\text { Gifuncionales } \\
\text { Semidiversificadas } \\
\text { Diversificadas }\end{array}$ & $\begin{array}{r}2 \\
6 \\
14 \\
15\end{array}$ & $\begin{array}{r}5.4 \\
16.2 \\
37.9 \\
40.5\end{array}$ & $\begin{array}{r}2 \\
8 \\
13 \\
14\end{array}$ & $\begin{array}{r}5.4 \\
21.6 \\
35.1 \\
37.9\end{array}$ & $\begin{array}{r}2 \\
1 \\
20 \\
14\end{array}$ & $\begin{array}{r}5 \cdot 1 \\
2.7 \\
5.7 \\
3 ? .7\end{array}$ \\
\hline
\end{tabular}

Fuente: cuadro 1.

Del cuadro 2 se puede concluir que existe una relativa estabilidad en la estructura de la PEA de las ciudades respecto a la del país. Sin embargo, al seguirle la pista a cada ciudad de 1940 a 1960 , se observan cambios importantes ocasionados por las modificaciones en la dinámica de una o varias ramas de actividad de las ciudades con respecto a la del país (cuadro 1 ).

El que las ciudades pierdan o ganen funciones predominantes implica que las ramas de actividad respectivas han tenido un crecimiento inferior o superior al nacional, respectivamente. Ello puede suceder debido a un elevado crecimiento industrial o a la existencia de abundantes industrias intensivas en mano de obra (como en el caso de la ciudad de León); diversificación y especialización de servicios (ciudad de México); extracción de gas y petróleo; intensa construcción de obras públicas, etc. Por ejemplo, entre 1940 y 1950, Tijuana, Guadalajara y Hermosillo, ciudades semidiversificadas al principio del período, se transformaron, al final del decenio, en diversificadas. El incremento en el comercio de Guadalajara, la construcción en Tijuana y la electricidad en Hermosillo, con respecto a los observados en el país en su conjunto, explican tal cambio.

Conceptualmente debería esperarse alguna relación entre la diversificación económica de las ciudades y su tamaño y tasa de crecimiento. Se piensa, teóricamente, que la diversificación de actividades es un indice positivo de la dinámica económica de las ciudades y que va aso-

valor de la producción generada en cada una de las ramas de actividad. Sin embargo, no se cuenta con la información necesaria para intentarla. 
ciada con economías de escala y externas. No obstante, en este estudio no se encontró relación alguna entre el grado de diversificación y el tamaño de las ciudades.

En cuanto a la tasa de crecimiento, tampoco se puede señalar un patrón definido para las ciudades unifuncionales y bifuncionales. Respecto a las semidiversificadas, 7 y 8 ciudades de 14 y 13 que había en 1940 y 1950, respectivamente, registraron incrementos medios anuales de $3.9 \%$ y menos. Por otro lado 8 y 9 de las ciudades diversificadas en 1940 y 1950 (de 14 y 15 respectivamente), presentaron incrementos medios anuales de entre $4.0 \%$ y $7.6 \%$.

Esto último no presenta un patrón definido de comportamiento, pero pone en duda el planteamiento teórico anterior. Una posible explicación está en que las ciudades menos diversificadas presentan mayor crecimiento demográfico dado que tienen sectores poco desarrollados, capaces de absorber considerables cantidades de mano de obra durante su proceso de crecimiento.

La primera función predominante. Se sostiene que, por su misma naturaleza, una ciudad debe tener características multifuncionales, en el sentido de poseer PEA dedicada a distintas actividades. Sin embargo, al comparar la PEA local con otra, en este caso la nacional, se observa que algunas ciudades se especializan en determinadas ramas. En esto último se basa la clasificación de carácter multifuncional del presente estudio, la cual tiene la desventaja, a pesar de ser más realista que las unifuncionales, de dificultar el análisis de la propia clasificación y de las características de la población. Por este motivo se realizó el estudio con base en la primera función predominante. Implícitamente se reconoce que ésta es la más importante, no obstante lo cual se considera conveniente extender el análisis a las funciones restantes.

\section{Cuadro 3}

MÉXICO: NÚMERO Y PORCIENTO DE CIUDADES SEGÚN LA PRIMERA FUNCIÓN PREDOMINANTE, 1940-1960

\begin{tabular}{|c|c|c|c|c|c|c|c|}
\hline \multirow{2}{*}{$\begin{array}{l}\text { Primera función } \\
\text { predominante en: }\end{array}$} & & \multicolumn{2}{|c|}{1940} & \multicolumn{2}{|c|}{1950} & \multicolumn{2}{|c|}{1960} \\
\hline & & Nứm. & $\%$ & Nüm. & $\%$ & Núm. & $\%$ \\
\hline $\begin{array}{l}\text { Mineria } \\
\text { Industria } \\
\text { construcción } \\
\text { Electricidad } \\
\text { Comercio } \\
\text { Transportes } \\
\text { Servicios }\end{array}$ & $\begin{array}{l}\text { (M) } \\
\text { (I) } \\
\text { (R) } \\
\text { (E) } \\
(\mathrm{C}) \\
\text { (T) } \\
(\mathrm{S})\end{array}$ & $\begin{array}{r}2 \\
8 \\
1 \\
0 \\
5 \\
9 \\
12\end{array}$ & $\begin{array}{r}5.4 \\
21.6 \\
2.7 \\
0.0 \\
13.5 \\
24.3 \\
32.4\end{array}$ & $\begin{array}{r}2 \\
6 \\
2 \\
0 \\
7 \\
7 \\
13\end{array}$ & $\begin{array}{r}5.4 \\
16.2 \\
5.4 \\
0.0 \\
18.9 \\
18.9 \\
35.2\end{array}$ & $\begin{array}{r}1 \\
8 \\
3 \\
0 \\
8 \\
4 \\
13\end{array}$ & $\begin{array}{r}2.7 \\
21.6 \\
8.1 \\
0.0 \\
21.6 \\
10.8 \\
35.2\end{array}$ \\
\hline
\end{tabular}

Fuente: cuadro 1.

La incipiente industrialización de México y, en particular, la gran concentración de la industria en unas cuantas ciudades, conduce a reflexionar acerca de cuáles actividades, por ser las predominantes, son las que en mayor o menor grado sostienen el desarrollo de la población de las ciudades. Éstas son las actividades no-agrícolas: los servi- 
cios y el comercio, ligados al sector gobierno y a la iniciativa privada. Así, el $32.4 \%$ en 1940 y en 1950 y 1960 el $35.2 \%$ de las ciudades tenían como actividad predominante los servicios (cuadro 3 ). Los servicios gubernamentales, financieros, profesionales, culturales, recreativos, domésticos y, además, el subempleo a que algunas de estas actividades dan cabida, satisfacen las necesidades de una creciente población urbana.

Las 12 ciudades en 1940 y 13 en 1950 y 1960 que tuvieron a los? servicios como primera función predominante (cuadro 1) son, en su gran mayoría, capitales de entidad: 10 en 1940, 12 en 1950 y 10 en 1960. Las ciudades restantes, de la frontera norte, son conocidas por la diversidad de servicios recreativo-turísticos que satisfacen la demanda anual de millones de norteamericanos.

Siguen en importancia relativa las ciudades que predominan en transportes: 9 en 1940 y 4 en 1960. Al respecto se señalan dos hechos importantes: primero, que con excepción de Villahermosa y Veracruz en 1940, las 7 ciudades restantes están en la zona norte o centro-norte del país, lo cual es indicio de la dirección hacia los Estados Unidos que sigue el comercio nacional. Segundo, que ciudades que tradicionalmente han sido centros de transporte, como Aguascalientes, Saltillo y Chihuahua, han perdido importancia relativa respecto al país. En contraste han surgido otros centros ferrocarrileros y puertos como Coatzacoalcos, Guaymas y Ensenada.

La importancia relativa de las ciudades industriales declinó de 1940 a 1950 y se recuperó durante el decenio siguiente. Las ciudades de este tipo, al principio y final del período, casi fueron las mismas: León, Guadalajara, Monterrey, Puebla, Irapuato, Aguascalientes, Querétaro y Orizaba. La disminución de 8 a 6 ciudades en el primer decenio (véase el cuadro 3 ) se debió, entre otras razones, a la acelerada industrialización del país y a su concentración en el área urbana de la ciudad de México, así como al escaso desarrollo industrial de Oaxaca e Irapuato. De 1950 a 1960, el país sufrió un descenso en el ritmo de industrialización mientras que Irapuato, Aguascalientes y Tampico (debido a un incremento de la refinación del petróleo), mostraron una mejoría en dicha actividad.

Las ciudades en donde predominaban el comercio y la construcción tuvieron auge durante todo el período. Las primeras, probablemente relacionadas con la comercialización de productos agropecuarios tanto para exportación como para cubrir la demanda interna ocasionada por el crecimiento y concentración de la población. Las segundas correspondieron a ciudades de rápido crecimiento demográfico, indicación indirecta de una dinámica económica más vigorosa que conduce, por lo general, a una mayor actividad constructora de los sectores público $\mathrm{y}$ privado.

Por último, en Pachuca y Tampico (incluyendo a Cd. Madero) predominaron las actividades extractivas en 1940 y 1950 . En el primer caso en minería la cual, a pesar de su decadencia, sigue siendo la forma de vida económica principal de esa ciudad, y en el segundo, la extracción de petróleo. Esta actividad dejó de ser predominante en Tampico, en 1960, y surgió la industria de transformación en primer término, debido 


\section{Cuadro 4}

MÉXICO: GRUPOS DE CIUDADES SEGÚN TAMAÑ̃ DE POBLACIÓN Y PRIMERA FUNCIÓN PREDOMINANTE, 1940-1960

(Porcientos horizontales)

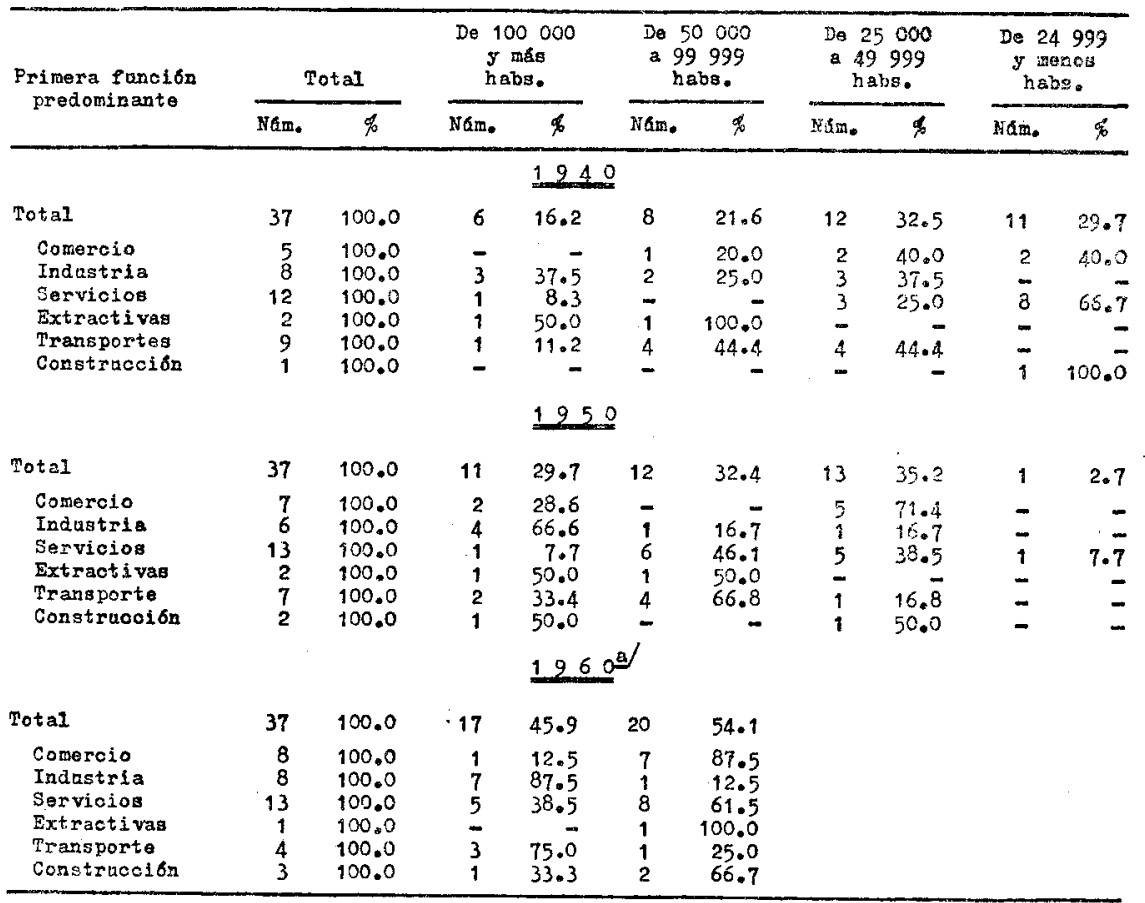

Fuente: cuadro 1.

a En 1960, las 37 ciudades fueron de 50000 y más habitantes.

a una reclasificación de la PEA de la actividad extractiva a la de transformación.

\section{Características de las cIUdAdes SEgúN SU ESPECIALIZación}

En esta parte del trabajo se analizan las tasas de crecimiento, tamaños y ubicación geográfica de las ciudades en relación con su especialización económica obtenida con base en la clasificación funcional. Se tratará de aclarar la hipótesis planteada en un estudio previo, ${ }^{12}$ según la cual las ciudades de menor tamaño deben su crecimiento demográîico a su condición de centros agrícolas y a las actividades comerciales y. de servicios relacionadas con ellas, mientras que, en determinada etapa de su crecimiento, el tamaño de su población les permite desarrollar un mercado interno que propicie la actividad industrial.

12 Unikel y Torres, op. cit. 
Primera función predominante y tamaño de la población. E1 cuadro 4 señala con claridad que, en el período 1940-1960, las ciudades comerciales y de servicios se concentraban en los intervalos menores de 50000 habitantes. Por otro lado, las ciudades industriales eran las de mayor tamaño de población, con la importante excepción de la de Méxi$\mathrm{co}$, especializada en servicios, no obstante generar (en su zona metropolitana) el $42.8 \%$ del valor de producción industrial del país.

La probable explicación de este fenómeno estriba en la interrelación de múltiples factores tales como la política de industrialización basada en la sustitución de importaciones; el creciente "efecto demostración" cuya cónsecuencia ha sido el aumento del consumo suntuario de grupos socioeconómicos minoritarios que residen en las grandes ciudades del país; la política tarifaria de los ferrocarriles que abarata el transporte de bienes primarios con respecto a los productos terminados; el creciente mercado constituido por la población de bajos ingresos para una industria que no puede desarrollarse en ciudades pequeñas; las razones psicosociales de los industriales y de sus familiares que los conduce a establecerse en las grandes ciudades; la concentración de las inversiones extranjeras en donde pueden contar con mayor seguridad y posibilidad de éxito así como estar cerca de los centros de decisión, de financiamiento, etcétera.

Existen dos tipos de ciudades especializadas en transporte. En primer lugar, algunas ciudades capitales, fronterizas o del interior, dedicadas al transporte de productos agropecuarios e industriales para el mercado interno y externo (Saltillo, Torreón, San Luis Potosí, Chihuahua y Nuevo Laredo) y en segundo, las ciudades portuarias (Veracruz y Mazatlán ). ${ }^{13}$ Estos dos tipos generalmente son de tamaño medio, entre 50000 y 100000 habitantes, hasta 1950. En 1960, tres de las cuatro ciudades de transporte superaban los 100000 habitantes, seguramente por el desarrollo de actividades adicionales al transporte: turismo en Mazatlán y Veracruz; servicios y comercio en Torreón y San Luis Potosí.

Por último, en las ciudades especializadas en industrias extractivas y de construcción no se encontró patrón alguno en relación con su tamaño de población.

Primera función predominante y localización geográfica. Se considera que la localización geográfica de las ciudades se basa tanto en factores ecológicos como socioeconómicos, y que tales circunstancias tienen asociación con un determinado tipo de especialización económica. A este respecto se esperaría, por ejemplo, que las ciudades portuarias, dado el papel que desempeñan en el transporte del grueso de las mercancías que se comercian con el exterior (excepto en las importaciones de los Estados Unidos), se especializaran en transportes y que la centralización del poder público de las entidades federales en su capital condujera a una especialización en servicios. Si se agrupan las 37 ciudades en cuatro tipos: 23 ciudades capitales federales, incluyendo a la ciudad de México, 6 ciudades fronterizas con los Estados

13 El área urbana de Tampico (que incluye a Cd. Madero) tuvo como segunda función predominante, de 1940 a 1960 , a los transportes. Véase el cuadro 1. 
Unidos, 3 ciudades portuarias y 6 ciudades del interior de la República (cuadro 1), se observa con claridad que aproximadamente el $50 \%$ de las capitales se especializaron en servicios (cuadro 5). Las capitales restantes lo hacían en transportes e industria de transformación en 1940 y 1950, así como en comercio e industria en 1960.

\section{Cuadro 5}

MÉxico: PRIMERa FunCión PREDOMINANTE POR CIUDADES SEGÚN SU LOCALIZACIÓN GEOGRÁFICA, 1940-1960

\begin{tabular}{|c|c|c|c|c|c|c|c|c|}
\hline \multirow{3}{*}{$\begin{array}{l}\text { Primera función } \\
\text { predominante }\end{array}$} & \multicolumn{8}{|c|}{ Ciudades } \\
\hline & \multicolumn{2}{|c|}{ Capitales } & \multicolumn{2}{|c|}{ Fronterizasa] } & \multicolumn{2}{|c|}{ Portuarias } & \multicolumn{2}{|c|}{ Del interior } \\
\hline & Nurm. & $\%$ & Núm. & $\%$ & Núm. & $\%$ & Vúm. & $\%$ \\
\hline \multicolumn{9}{|c|}{1940} \\
\hline Total & 23 & 100.0 & 6 & 100.0 & 3 & 100.0 & 6 & 100.0 \\
\hline $\begin{array}{l}\text { Comercio } \\
\text { Industria } \\
\text { Servicios } \\
\text { Extractivas } \\
\text { Transporte } \\
\text { Construcción }\end{array}$ & $\begin{array}{r}2 \\
5 \\
10 \\
1 \\
5 \\
-\end{array}$ & $\begin{array}{r}8.7 \\
21.7 \\
43.5 \\
4.4 \\
21.7 \\
- \\
1\end{array}$ & $\frac{2}{3}$ & $\begin{array}{r}33.0 \\
50.0 \\
17.0 \\
-\end{array}$ & $\begin{array}{l}- \\
\overline{-} \\
\frac{1}{2} \\
-\end{array}$ & $\begin{array}{r}- \\
33.0 \\
67.0 \\
-\end{array}$ & $\begin{array}{l}1 \\
3 \\
-x+4 \\
-1 \\
1\end{array}$ & $\begin{array}{r}16.7 \\
50.0 \\
-5 \\
16.7 \\
16.7\end{array}$ \\
\hline Total & 23 & 100.0 & 6 & 100.0 & 3 & 100.0 & 6 & 100.0 \\
\hline $\begin{array}{l}\text { Comercio } \\
\text { Industria } \\
\text { Servicies } \\
\text { Extractivas } \\
\text { Transporte } \\
\text { Construcción }\end{array}$ & $\begin{array}{r}2 \\
4 \\
12 \\
1 \\
4 \\
-\end{array}$ & $\begin{array}{r}8.7 \\
17.4 \\
52.1 \\
4.4 \\
17.4 \\
-2\end{array}$ & $\begin{array}{l}2 \\
\overline{2} \\
\overline{1} \\
1\end{array}$ & $\begin{array}{l}33.0 \\
33.0 \\
17.0 \\
17.0\end{array}$ & $\begin{array}{l}= \\
\overline{1} \\
z\end{array}$ & $\begin{array}{r}= \\
67.0 \\
-\end{array}$ & $\begin{array}{l}3 \\
2 \\
- \\
- \\
-\end{array}$ & $\begin{array}{r}50.0 \\
33.0 \\
- \\
=-\end{array}$ \\
\hline Total & 23 & 100.0 & 6 & 100.0 & 3 & 100.0 & 6 & 100.0 \\
\hline $\begin{array}{l}\text { Comercio } \\
\text { Industria } \\
\text { Servicios } \\
\text { Extractivas } \\
\text { Transporte } \\
\text { Construcción }\end{array}$ & $\begin{array}{r}4 \\
4 \\
10 \\
1 \\
1 \\
3\end{array}$ & $\begin{array}{r}17.4 \\
17.4 \\
43.5 \\
4.3 \\
4.3 \\
13.1\end{array}$ & $\begin{array}{l}2 \\
- \\
- \\
-\end{array}$ & $\begin{array}{r}33.0 \\
67.0 \\
=\end{array}$ & $\begin{array}{l}\overline{1} \\
\bar{z} \\
\bar{z}\end{array}$ & $\begin{array}{r}33 . \overline{-} \\
67 . \overline{0} \\
-\end{array}$ & $\begin{array}{l}2 \\
3 \\
- \\
- \\
1 \\
-\end{array}$ & $\begin{array}{r}33.0 \\
50.0 \\
- \\
17.0 \\
-\end{array}$ \\
\hline
\end{tabular}

Fuente: cuadro 1 .

a La ciudad de Mexicali se incluye tanto en el grupo de las ciudades capitales como en el de las fronterizas.

El reducido personal del aparato político-administrativo estatal, aun incluyendo el federal, comparado con aquel con que cuenta la capital de la República, así como el que existe en otro tipo de servicios (profesionales, educativos, médicos, etc.), puede ser motivo importante por el cual en estas ciudades capitales no predominen los servicios.

- Las ciudades fronterizas se especializaban principalmente en servicios y comercio; aunque aquéllos son distintos de los que prestan las capitales. Los servicios son, en su mayoría, turístico-recreativos no obstante que parte de ellos se orientan a satisfacer el sector agrícola moderno (en un solo caso de los seis estudiados, úna ciudad fronteriza es también capital: Mexicali). El comercio es abundante, también en relación con el turismo, pero de igual manera es importante el renglón de salida y entrada de mercancías. De hecho, algunas ciudades de la 
frontera norte, como Nuevo Laredo y Matamoros, son puertos terrestres tan importantes como los fluviales, debido a la carga que pasa por dichos puntos, de y hacia Estados Unidos, principal país con el que México comercia. En ciudades del interior de la región noroeste sobresale la importancia del sector terciario y su íntima conexión con la actividad agropecuaria. Ciudades como Hermosillo, Ciudad Obregón y Culiacán son claros ejemplos de este hecho.

Las ciudades portuarias (Mazatlán, Veracruz y Tampico) se especializan fundamentalmente en transportes. Sólo en Tampico esta rama no constituyó la primera función predominante; las grandes instalaciones de PEMEX en Ciudad Madero hicieron que en 1940 y 1950 predominara la industria extractiva y en 1960, la de transformación, tal como se dijo arriba. Sin embargo, transportes ocupó el segundo lugar (cuadro 1).

Finalmente, dentro del grupo de ciudades del interior, la mitad de ellas (Irapuato, León y Orizaba) tenían como función predominante la industria de transformación, en 1940 y 1950 ; en 1950 sólo las dos últimas mantuvieron esta especialización. Fueron comerciales Celaya en todo el período estudiado, Torreón e Irapuato en 1950, y Ciudad Obregón en 1960.

Primera función predominante y ritmo de crecimiento demográfico. Considerando que las diferencias en la dinámica de cambio de las ramas de actividad influyen en el ritmo de crecimiento de las ciuda-

\section{Cuadro 6}

MÉXICO: CIUDADES SEGÚN LA PRIMERA FUNCIÓN PREDOMINANTE E INCREMENTO MEDTO ANUAL DE CRECIMTENTO DE SU POBLACIÓN, 1940 Y 1950

(Porcientos horizontales)

\begin{tabular}{|c|c|c|c|c|c|c|c|c|}
\hline \multirow{3}{*}{$\begin{array}{l}\text { Primera funcion } \\
\text { predominante }\end{array}$} & \multirow{3}{*}{$\begin{array}{l}\text { Nánero } \\
\text { ciudades }\end{array}$} & \multirow{3}{*}{$\not$} & \multicolumn{6}{|c|}{ Incremento medio anual de poblacion } \\
\hline & & & \multicolumn{2}{|c|}{$7.5 \%$ y más } & \multicolumn{2}{|c|}{ De 4.0 a $7.4 \%$} & \multicolumn{2}{|c|}{$3.9 \%$ y menos } \\
\hline & & & Nam. & $\%$ & vám. & \& & Nám. & $\not q$ \\
\hline \multicolumn{3}{|c|}{1940} & \multicolumn{6}{|c|}{ 1940-1950 } \\
\hline $\begin{array}{l}\text { Servicios } \\
\text { Transportes } \\
\text { Industria } \\
\text { Comerolo } \\
\text { Extractiva } \\
\text { Construcoion }\end{array}$ & $\begin{array}{r}12 \\
9 \\
8 \\
5 \\
2 \\
1\end{array}$ & $\begin{array}{l}100.0 \\
100.0 \\
100.0 \\
100.0 \\
100.0 \\
100.0\end{array}$ & $\frac{6}{2}$ & $\begin{array}{r}50.0 \\
40 . \overline{-} \\
100 . \overline{0}\end{array}$ & $\begin{array}{l}2 \\
4 \\
6 \\
2 \\
- \\
-\end{array}$ & $\begin{array}{r}16.7 \\
44.4 \\
75.0 \\
40.0 \\
= \\
=\end{array}$ & $\begin{array}{l}4 \\
5 \\
2 \\
1 \\
2 \\
-\end{array}$ & $\begin{array}{r}33.3 \\
55.6 \\
25.0 \\
20.0 \\
100.0 \\
-\end{array}$ \\
\hline \multicolumn{3}{|c|}{1950} & \multicolumn{6}{|c|}{$1250-1260$} \\
\hline $\begin{array}{l}\text { Servicios } \\
\text { Transportes } \\
\text { Indastria } \\
\text { Comercio } \\
\text { Extractiva } \\
\text { Constraccion }\end{array}$ & $\begin{array}{r}13 \\
7 \\
6 \\
7 \\
2 \\
2\end{array}$ & $\begin{array}{l}100.0 \\
100.0 \\
100.0 \\
100.0 \\
100.0 \\
100.0\end{array}$ & $\begin{array}{l}4 \\
- \\
- \\
-\end{array}$ & $\begin{array}{r}30.8 \\
= \\
= \\
50 . \overline{0}\end{array}$ & $\begin{array}{l}7 \\
3 \\
3 \\
5 \\
1\end{array}$ & $\begin{array}{r}53.8 \\
42.9 \\
50.0 \\
71.4 \\
50 . \overline{0}\end{array}$ & $\begin{array}{l}2 \\
4 \\
3 \\
2 \\
2 \\
-\end{array}$ & $\begin{array}{r}15.4 \\
57.1 \\
50.0 \\
28.6 \\
100.0 \\
-\end{array}$ \\
\hline
\end{tabular}

Fuente: Las tasas de crecimiento, en L. Unikel, "El proceso de urbanización en México: distribución y crecimiento de la población urbana", Demografía y EconoMía, Vol. II, Núm. 2, El Colegio de México, cuadro 20. El tipo de actividad de las ciudades se obtuvo del cuadro 1. 
des, surge la siguiente pregunta: ¿depende el ritmo de crecimiento de las ciudades de la rama de actividad en que se especializan?

Habiendo agrupado a las ciudades según su tasa anual de crecimiento en tres clases arbitrarias: $a$ ) de crecimiento rápido, aquéllas con $7.5 \%$ y más; $b$ ) de crecimiento medio, las que registraron tasas entre $4.0 \%$ y $7.5 \%$; y c) de crecimiento bajo, con tasas menores al $3.9 \%$, se encontró lo siguiente : en el decenio 1940-1950, de las 12 ciudades con predominio en servicios, 8 registraron incrementos medios anuales superiores al $4 \%$ anual de las cuales 6 estuvieron por arriba del $7.5 \%$ (cuadro 6). Del mismo modo, 6 de las ciudades de rápido crecimiento se especializaban en servicios.

De 1950 a 1960, sólo 4 de las 13 ciudades especializadas en servicios presentaron un crecimiento rápido y 7 más un incremento medio. Además, de las 5 ciudades de crecimiento rápido 4 correspondieron a la función predominante servicios (cuadro 7).

En ambos períodos, ninguna ciudad especializada en las ramas de transporte, industria de transformación e industria extractiva acusó crecimiento rápido. Las especializadas en transportes experimentaron, en general, tasas de crecimiento bajo, mientras que la mayoría de las industriales fueron de crecimiento medio. Las ciudades con predominio de las actividades extractivas registraron rechazo de población, o sea, un saldo neto migratorio negativo; los casos de Pachuca y Tam-

\section{Cuadro 7}

MÉxico: CiUdAdES SEgúN EL INCREMENTo MEDIO ANUAL DE SU POBLACión Y PRIMERA FUNCIÓN PREDOMINANTE, 1940-1950 Y 1950-1960

(Porcentajes verticales)

\begin{tabular}{|c|c|c|c|c|}
\hline \multirow{2}{*}{$\begin{array}{l}\text { Tipo de ciudades segín } \\
\text { el incremento medio } \\
\text { anual de la población }\end{array}$} & \multicolumn{2}{|c|}{$1940-1950$} & \multicolumn{2}{|c|}{$1950-1960}$. \\
\hline & $\begin{array}{l}\text { Núm. de } \\
\text { ciudades }\end{array}$ & $\not$ & $\begin{array}{l}\text { Núm. de } \\
\text { ciudades }\end{array}$ & \% \\
\hline De $7.5 \%$ y nás & 9 & 100.0 & 5 & 100.0 \\
\hline $\begin{array}{l}\text { Servicios } \\
\text { Construcción } \\
\text { Conercio }\end{array}$ & $\begin{array}{l}6 \\
1 \\
2\end{array}$ & $\begin{array}{l}66.7 \\
11.1 \\
22.2\end{array}$ & $\begin{array}{l}4 \\
1 \\
-\end{array}$ & $\begin{array}{r}80.0 \\
20.0 \\
-\quad-\end{array}$ \\
\hline De $4.0 \%$ a $7.4 \%$ & 14 & 100.0 & $\underline{29}$ & 100.0 \\
\hline $\begin{array}{l}\text { Servicios } \\
\text { Transporte } \\
\text { Constmicoión } \\
\text { Ircustria } \\
\text { Comercio }\end{array}$ & $\begin{array}{l}2 \\
\frac{4}{6} \\
5 \\
2\end{array}$ & $\begin{array}{r}14.3 \\
28.6 \\
42 . \overline{8} \\
14.3\end{array}$ & $\begin{array}{l}7 \\
3 \\
3 \\
2 \\
2 \\
5\end{array}$ & $\begin{array}{l}36.9 \\
15.8 \\
10.5 \\
19.5 \\
26.3\end{array}$ \\
\hline A, 00 y menos & 14 & 100.0 & 13 & 100.0 \\
\hline $\begin{array}{l}\text { Irmaporte } \\
\text { Comeveio } \\
\text { Industria } \\
\text { Jxtraotiva } \\
\text { Servicios }\end{array}$ & $\begin{array}{l}i_{5}^{4} \\
1 \\
3 \\
2 \\
4\end{array}$ & $\begin{array}{r}28.6 \\
7.1 \\
22.4 \\
1.4 .3 \\
28.6\end{array}$ & $\begin{array}{l}5 \\
2 \\
2 \\
2 \\
2\end{array}$ & $\begin{array}{l}33.4 \\
15.4 \\
15.4 \\
15.4 \\
15.4\end{array}$ \\
\hline
\end{tabular}

Fuente: Las tasas de crecimiento, en L. Unikel, "El proceso de urbanización en México: distribución y crecimiento de la población urbana", DEMografía Y EconoMía, Vol. II, Núm. 2, El Colegio de México, cuadro 20. El tipo de actividad de las ciudades se obtuvo del cuadro 1. 


\section{Cuadro 8}

México: Participación e INCREMENTOS MEdios DE LA PEA EN EL SEctor SECUNDARIO Y TERCIARIO, SEGÚN CIUDADES CON INCREMENTO MEDIO ANUAL DE $7.5 \%$ Y MÁs, 1940-1960

(Porcientos)

\begin{tabular}{|c|c|c|c|c|}
\hline \multirow{2}{*}{ Cladados } & \multicolumn{2}{|c|}{ Sector } & \multicolumn{2}{|c|}{ Tasa de } \\
\hline & terciariog & secundariob & terciarizacion & industriallzaoion \\
\hline & \multicolumn{2}{|c|}{1940} & \multicolumn{2}{|c|}{$1240-1950$} \\
\hline \multirow[t]{2}{*}{$\begin{array}{l}\text { Kexicali } \\
\text { Ti jusna } \\
\text { ce. Jakrez } \\
\text { Caliackn } \\
\text { Matsmoros } \\
\text { Reyrosa }\end{array}$} & $\begin{array}{l}54.6 \\
71.4 \\
57.4 \\
59.7 \\
57.5 \\
51.6\end{array}$ & $\begin{array}{l}21.6 \\
19.0 \\
26.8 \\
28.1 \\
21.2 \\
19.6\end{array}$ & $\begin{array}{r}2.17 \\
-\quad 24.70 \\
-\quad 3.90 \\
5.06 \\
5.41 \\
15.38\end{array}$ & $\begin{array}{r}21.8 \\
50.2 \\
29.2 \\
8.7 \\
21.6 \\
37.2\end{array}$ \\
\hline & \multicolumn{2}{|c|}{1950} & \multicolumn{2}{|c|}{$1950-1960$} \\
\hline $\begin{array}{l}\text { Texicali } \\
\text { mijuana } \\
\text { Tepio } \\
\text { cd. Obregon }\end{array}$ & $\begin{array}{l}55.8 \\
55.7 \\
55.1 \\
50.1\end{array}$ & $\begin{array}{l}26.9 \\
31.5 \\
36.1 \\
42.4\end{array}$ & $\begin{array}{r}2.6 \\
15.8 \\
-10.9 \\
21.0\end{array}$ & $\begin{array}{r}6.6 \\
6.7 \\
-\quad 9.9 \\
-33.5\end{array}$ \\
\hline
\end{tabular}

Fuentes: cuadros A1, A2 y A3.

a Comprende comercio, comunicaciones y transportes y servicios.

b Comprende industria extractiva, de transformación, construcción y electricidad y gas.

pico así lo ejemplifican. ${ }^{14}$ Las ciudades especializadas en comercio y construcción presentaron por lo general un crecimiento demográfico rápido y medio.

Como respuesta a la pregunta inicial de este inciso se concluye que existe cierta relación entre las ciudades especializadas en servicios y sus altas tasas de crecimiento demográfico. Se puede señalar que las ciudades industriales y comerciales poseen, en general, tasas de crecimiento medio pero son, sin embargo, de atracción. ${ }^{25}$ Por último, las ciudades especializadas en transportes e industrias extractivas son de crecimiento bajo.

La elevada tasa de crecimiento de las ciudades especializadas en servicios permite suponer que ello conduce a una presión sobre el empleo, originando un aumento relativo del sector terciario (proceso de terciarización) y, seguramente, la presencia de grupos subocupados y desempleados, en particular de población migrante.

En el cuadro 8 se observa que, en el período 1940-1950, de las seis ciudades de crecimiento rápido, cuatro se terciarizaron. Sin embargo, las tasas de crecimiento del sector secundario fueron muy superiores a las del terciario, lo cual hace suponer que se produjo una transferencia de mano de obra del sector terciario al secundario; esto presupone que la PEA que migra del campo a estas ciudades se alojó, principalmente, en el sector terciario. También se prueba con ello que, no obs-

14 Véase L. Unikel, "E1 proceso de urbanización en México; distribución y crecimiento de la población", Demografía Y ECoNomía, Vol. II, Núm. 2, pp. 115-156.

15 Tienen un saldo neto migratorio positivo, ibid., pp. 151-152. 
tante que estas ciudades se especializan en servicios, el sector más dinámico fue el industrial y que entre ambos absorbieron los grandes volúmenes de PEA que resultaron de las elevadas tasas de crecimiento demográfico.

Lo anterior sólo se presenta en 1950-1960 en Mexicali, mientras que en Tijuana y Ciudad Obregón fue más dinámico el proceso de terciarización. Es indudable que el sector terciario de estas dos ciudades absorbió la creciente PEA conforme al supuesto de subocupación y desempleo antes mencionado.

\section{GRADO DE ESPECIALIZACIÓN INTERNA}

Los estudios acerca de la especialización económica de las ciudades generalmente han sido de naturaleza cualitativa (según tipos) y en contadas ocasiones según el grado de especialización (cuantivamente) ${ }^{16}$ Con objeto de completar el análisis de la clasificación funcional (comparación ciudad-país), con el de la estructura interna de la PEA de las ciudades, se ha utilizado un índice de diferenciación económica que se denominará IED. Éste varía entre 0 y 1 según que la estructura de la PEA se distribuya por partes iguales o se concentre totalmente en una sola rama de actividad, respectivamente (véase el apéndice).

\section{Cuadro 9}

MÉXICO: ÍNDICE DE DIFERENCIACIÓN ECONÓMICA (IED) a SEGÚN LA PRIMERA FUNCIÓN PREDOMINANTE, POR TIPOS DE CIUDADES, 1940-1960

\begin{tabular}{lccc}
\hline $\begin{array}{l}\text { Tipo de } \\
\text { ciudades }\end{array}$ & 1940 & 1950 & 1960 \\
\hline Industriales & 0.165 & 0.165 & 0.150 \\
De servicios & 0.157 & 0.126 & 0.131 \\
Comerciales & 0.131 & 0.111 & 0.116 \\
De construcción & 0.115 & 0.098 & 0.109 \\
De transportes & 0.101 & 0.102 & 0.102 \\
Mineras & 0.084 & 0.063 & 0.082 \\
\hline
\end{tabular}

Fuentes: cuadros 1 y A4.

a Véase el apéndice.

IED y tipos de ciudades. Un análisis somero del IED de las 37 ciudades en el período 1940-1960 indica que las ciudades con predominio industrial son las que concentran más PEA en una o dos ramas de actividad; le siguen en orden decreciente, las de servicios, comerciales, de construcción, de transportes y minería (cuadro 9). Dado que el porciento de PEA industrial en las ciudades en las cuales predomina

I6 Por ejemplo la clasificación del presente estudio es de naturaleza cualitativa, pues no diferencia grados de especialización entre ciudades con las mismas funciones predominantes (véase cuadro 1). Por otro lado, un reducido número de clasificaciones intenta cierto grado de jerarquización de funciones mediante el empleo de un score estadístico. Tal es el caso de la clasificación multifuncional de Nelson que utiliza la desviación estándar para tal objeto, dando por resultado ciudades industriales, mineras, de servicios, etc., más una, dos o tres desviaciones estándar. Para mayores detalles, Véase H. Nelson, "A Service Classification of American Cities", Economic Geography, Vol. 31, 1955, pp. 189-210. 
esta rama de actividad es similar al de servicios en aquellas con predominio en esta rama, la mayor concentración de PEA en las primeras se tendría que explicar por la actividad que posee el segundo valor relativo. Efectivamente, en los tres años estudiados se encontró que la segunda rama de actividad de la mayoría de las ciudades industriales -excepto dos en 1940 y una en 1950 y 1960 - es la de servicios. También se observó que la suma de las dos primeras actividades en las ciudades industriales es mayor, en general, que la de las ciudades de servicios.

El hecho de que las ciudades industriales sean las que poseen mayor grado de especialización es un fenómeno paralelo al de la concentración industrial en algunas ciudades. Así, el porciento promedio de PEA industrial de las 37 ciudades respecto al total nacional pasó de $24.5 \%$ en 1940 a $34.5 \%$ en 1960 , lo cual muestra la creciente concentración industrial en las principales ciudades y en particular en las de México y Monterrey.

Es importante señalar que el mayor grado de especialización en las ciudades industriales no es un fenómeno general que se observe en otros países. En Estados Unidos, por ejemplo, se encontró que en 1950 las ciudades comerciales registraron un mayor grado de especialización que las industriales, ${ }^{17}$ lo cual refleja las diferencias existentes en las estructuras productivas de ambos países.

IED y tamaños de ciudades. ¿Las ciudades grandes tienen más diversificada o más concentrada sur actividad económica? Teóricamente se esperaría que las ciudades de mayor tamaño estuvieran más diversificadas, debido a que tienden a una mayor autosuficiencia y a desarrollar diversas actividades, dado que la magnitud de su población proporciona el mercado necesario para absorber, en mayor medida, su producción.

\section{Cuadro 10}

MÉXico: ÍNDICE DE DIFERENCIACTóN ECONÓMICA (IED) POR taMaÑos DE CIUDADES, $1940-1960$

\begin{tabular}{|c|c|c|c|}
\hline \multirow{2}{*}{ Tamaños de ciudades } & \multicolumn{3}{|c|}{$I \pm D^{a /}$} \\
\hline & 1940 & 1950 & 1960 \\
\hline $\begin{array}{l}\text { De } 100 \text { 000 y mÁs habitantes } \\
\text { De } 50 \text { 000 a } 99999 \text { habitantes } \\
\text { De } 25000 \text { a } 49999 \text { habitantes } \\
\text { De } 24 \quad 999 \text { y menos habitantes }\end{array}$ & $\begin{array}{l}0.116 \\
0.135 \\
0.136 \\
0.151\end{array}$ & $\begin{array}{l}0.125 \\
0.113 \\
0.118 \\
0.114\end{array}$ & $\begin{array}{r}0.132 \\
0.120 \\
-\end{array}$ \\
\hline
\end{tabular}

Fuentes: cuadros 1 y A4.

a Véase el apéndice.

Los datos del cuadro 10 confirman lo anterior para el año de 1940 , en el cual las ciudades más pequeñas tienen un IED mayor. Sin embargo, en 1950 y 1960 la situación se invirtió y, a medida que las ciudades

17 Eiji C. Amemiya, "Economic Differentiation and Social Organization of Standard Metropolitan Areas", Journal of Regional Science, Vol. 5, Núm. 2, 1964, p. 60. 
eran más grandes, su IED fue mayor. Además, sólo en las ciudades de 100000 y más habitantes el IED fue creciente. O sea, que en estos centros urbanos se apareció un aumento en el grado de especialización y por tanto en la concentración económica.

Una posible explicación de este fenómeno sería el cambio experimentado en las estructuras de la producción del país hacia los sectores no agrícolas y en particular el hecho de que las ciudades industriales son las que mayor concentración presentan en su PEA industrial $\mathrm{y}$ de servicios. Esta concentración o grado de especialización de la actividad económica en las grandes ciudades, constituye uno de los rasgos esenciales de los procesos de urbanización y desarrollo económico en el período 1940-1960.]

\section{Funciones económicas predominantes en 1970}

La clasificación funcional para 1970 se realizó con información censal a nivel municipal. La PEA por ramas de actividad económica presenta algunas modificaciones con respecto a la de 1960, lo cual dificulta su estudio comparativo. ${ }^{18}$ No obstante y de manera preliminar, es necesario extraer algunas inferencias sobre la clasificación funcional de los municipios que contienen a las 37 ciudades.

Independientemente de los ajustes que deben hacerse a las cifras de 1970 para obtener datos sobre las ciudades, la clasificación funcional aplicada en este trabajo se basa en cifras relativas ciudad-país o, en el caso de 1970, municipio-país. Además, las actividades económicas no-agrícolas de los municipios estudiados están altamente concentradas en las ciudades. Ambas consideraciones permiten suponer que, con las reservas del caso, la comparación 1960-1970 puede arrojar resultados menos sesgados de lo que las diferencias censales indican. También se ha supuesto para el análisis que la nueva rama de actividad "industria del petróleo" se incluía casi totalmente en la de "industria de transformación" en los años anteriores, con lo cual se sobrestima un poco esta rama y se subestima la de "industria extractiva". Por último, las "actividades insuficientemente especificadas" no predominaron en primer lugar en ninguno de los 37 casos (cuadro 11), por lo cual fueron excluidas del análisis. Establecidas las condiciones en que se elaboró la clasificación funcional para 1970, se considera que los resultados del análisis, a reserva de su confirmación con datos

18 Esta apreciación se funda en el documento preliminar de Clara J. de Bialostozky, "Clasificación de actividades económicas", Dirección General de Estadística, Oct. 1971 (mecanografiado). En vista de las modificaciones en algunos de Ios conceptos utilizados en la clasifícación censal de la PEA de 1970, se considera difícil lograr la total comparabilidad entre las cifras del último censo de población y las de los tres anteriores. Para lograr el máximo acercamiento al objetivo mencionado son necesarios tres ajustes: el primero exige el reagrupamiento de varios de los 91 grupos de actividad económica en que se subdividen las once grandes divisiones en que se clasificó la PEA de los municipios estudiados. El segundo requiere la redistribución de la "actividad insuficientemente especificada" entre las restantes. EI tercero implica convertir al nivel de ciudad la información municipal. Para los dos primeros ajustes es necesario consultar el documento de la Sra. Bialostozky. Para el tercero, véase Unikel y Torres, loc. cit., pp. $40-42$. 
más comparables a los de 1960, ofrecen una idea general de lo ócurrido en el último decenio. El cuadro 11 presenta los tipos de especialización de ciudades en 1970 .

Según el número de funciones predominantes de 1960 a 1970 , los cambios más destacados se produjeron en las ciudades (suponiendo la clasificación funcional de municipios en 1970 equivalente a la de las ciudades principales que contienen) semidiversificadas y diversificadas: éstas registraron un aumento sustancial (cuadros 2 y 12). Este fenómeno refleja la mayor dinámica de las nuevas ramas de actividad en las ciudades que se diversificaron, con respecto al país en su con-

\section{Cuadro 11}

MÉxico: Clasificación funcional de los MUNicipios oUe CONTIENEN A LAS PRINCIPALES CIUDADES, 1970

\begin{tabular}{|c|c|c|c|}
\hline Nunioipios & $\begin{array}{l}\text { Funciones pre } \\
\text { dominantes b] }\end{array}$ & Manicipiog & $\begin{array}{l}\text { Fanciones pre } \\
\text { dominantes b7 }\end{array}$ \\
\hline $\begin{array}{l}\text { Aguascalientea } \\
\text { Mericali } \\
\text { Mijaana } \\
\text { Saltillo } \\
\text { Torrebn } \\
\text { Cd. Juárez } \\
\text { Chihuahua } \\
\text { Cd. de Nexico } \\
\text { Darargo } \\
\text { Celaya } \\
\text { Irepuato } \\
\text { Ledn } \\
\text { Pachuca } \\
\text { Guadalajara } \\
\text { Toluca } \\
\text { Horelia } \\
\text { Cuernavaca } \\
\text { Tepio } \\
\text { Monterrey }\end{array}$ & $\begin{array}{l}T-6 \\
S \\
C-S K \\
I-T K M \\
C-T S E K \\
S-C K \\
S-T K O M E \\
S-I \\
S-T K C M \\
C-X S T \\
C-I T E S \\
I \\
M-S C S \\
I-X C T \\
X-S T R \\
S-K C E \\
S-K \\
S-C E T K \\
I-K T\end{array}$ & $\begin{array}{l}\text { Oaxaca } \\
\text { Puabla } \\
\text { Querstaro } \\
\text { San Luis Potost } \\
\text { Cultacin } \\
\text { Mazatlín } \\
\text { Cajeme c/ } \\
\text { Hermosillo } \\
\text { Centro c/ } \\
\text { Cd. Victoria } \\
\text { Katamoros } \\
\text { Nuevo Laredo } \\
\text { Reynosa } \\
\text { Tampico } \\
\text { Jalapa } \\
\text { Orizaba } \\
\text { Veracruz } \\
\text { Merida }\end{array}$ & $\begin{array}{l}\text { S-CTEK } \\
\text { I-CSET } \\
\text { I-KSE } \\
\text { T-KMC } \\
\text { S-CTK } \\
\text { S-TC } \\
\text { C-SKT } \\
\text { S-CKRT } \\
\text { S-CFKE } \\
\text { S-KE } \\
\text { C-KT } \\
\text { S-CTK } \\
\text { P-KC } \\
\text { P-SKCT } \\
\text { S-TKKE } \\
\text { I-TCE } \\
\text { S-TCPE } \\
\text { S-CIIE }\end{array}$ \\
\hline
\end{tabular}

Fuente: Cálculos elaborados según el índice de trabajadores excedentes presentado en el apéndice y con base en los datos del cuadro A4.

a La información manejada para 1970 es municipal, debido a la falta de tiempo para ajustar tales datos al nivel de ciudad. Sin embargo, la PEA no agrícola del municipio o municipios (cuando se trata de una área urbana que abarca dos o más) se concentra en muy elevada proporción en las ciudades, superando, en la mayoría de los casos, el $90 \%$

b S, servicios; I, industria de transformación; $\mathrm{C}$, comercio; $\mathrm{T}$, comunicaciones y transportes; $\mathrm{K}$, construcción; $\mathrm{E}$, electricidad $\mathrm{y}$ gas; $\mathrm{P}$, industria del petróleo; $M$, industrias extractivas. Se excluyeron las actividades no especificadas como función predominante en las 10 ciudades en que tal grupo se presentó como especializado. Se aclara que en ningún caso constituyó la primera función.

e Cajeme contiene a Cd. Obregón y el Centro a Villahermosa.

junto. Lo anterior indica que la estructura económica de cada ciudad se distribuye en mayor número de actividades, lo cual, teóricamente, debería fortalecer la dinámica interna de la ciudad y reducir su dependencia de los mercados externos, tanto en sus importaciones como en sus exportaciones; en suma, adquiriría mayor importancia su mercado interno. Sin embargo, puede también expresar una dinámica nacional del sector no agrícola más lenta que la de las ciudades que se han diversificado, o de un aumento en el subempleo, principalmente en las ramas del sector terciario. 
En vista de que el comportamiento de la PEA no agrícola depende en gran medida del que registran las 37 ciudades, es probable que las que se diversificaron no lo hicieron en respuesta a un crecimiento acelerado de las nuevas ramas de actividad predominantes, sino más bien a la menor lentitud con que absorbieron PEA con respecto a la media nacional. En cualquier caso, las ramas de actividad que surgieron en las nuevas ciudades diversificadas fueron, principalmente: servicios, comunicaciones y transportes, comercio y construcción; esto es, fundamentalmente, el sector terciario.

\section{Cuadro 12}

MÉXico: CiUdAdES SEGúN la PRIMERA FUNCIÓN PREDOMINANTE Y POR EL NÚMERO DE FUNCIONES, 1970

\begin{tabular}{lccccc}
\hline $\begin{array}{c}\text { Función } \\
\text { predominante }\end{array}$ & $\begin{array}{c}\text { Nime de } \\
\text { ciuduades }\end{array}$ & $\begin{array}{c}\text { Función } \\
\text { predowinante }\end{array}$ & $\begin{array}{c}\text { Nüm. de } \\
\text { ciudades }\end{array}$ & $\begin{array}{c}\text { Ciudades segín } \\
\text { número de funcioneg }\end{array}$ & $\begin{array}{c}\text { súm. de } \\
\text { ciudades }\end{array}$ \\
\hline Servicios & 18 & Petróleo & 2 & Unifuncionsles & 2 \\
Industria & 7 & Minería & 1 & Bifuncionales & 2 \\
Comercio & 6 & Construcción & 1 & Semidiversificadas & 8 \\
Transporte & 2 & Electricidad & 0 & Diversificadas & 25 \\
\hline
\end{tabular}

Fuente: cuadro 11.

a En el número de funciones no se consideró la función predominante en actividades no especificadas.

Respecto al tipo de especialización de las ciudades según la primera función predominante, se presentan cambios significativos de 1960 a 1970. En primer lugar, las ciudades especializadas en servicios aumentaron substancialmente: de 13 en 1960 a 18 en 1970 (cuadros 3 y 12). Esto refleja el fuerte crecimiento de la PEA en la rama de servicios, y puede significar que la tendencia, que empezó a manifestarse de 1950 a 1960 en cuanto a la terciarización dé las principales ciudades del país, se ha acentuado debido, en gran parte, a la aceleración de la migración campo-ciudad durante este decenio, y a que la creciente PEA ha tenido que refugiarse en el sector terciario en condiciones crecientes de subempleo y desempleo. En segundo, si se agregan las ciudades especializadas en industria del petróleo a las de transformación - tal como se encontraron en 1960 - se tienen en 1970 nueve ciudades industriales, esto es, tres más que en 1950 y una más que en 1960. Así, las ciudades industriales continúan aumentando según la tendencia observada desde 1950 aunque, de 1960 a 1970, más lentamente. Por último, como corolario de los aumentos de los tipos anteriores de ciudades se observa, en 1960-1970, una disminución de las ciudades de transporte, construcción y comercio, actividades señaladas como dinámicas en este período aunque no lo suficiente como para aparecer como primeras funciones predominantes.

- En resumen, el período $1960-1970$ presenta dos tendencias relativamente claras e importantes: mayor diversificación de las actividades económicas urbanas, lo cual implica mayor semejanza en la estructura ocupacional de las ciudades y el país, debido en gran medida a mayor concentración de las actividades no agrícolas en algunos de los 37 cen- 
tros urbanos; y aumento en el predominio de los servicios como primera función. Esto último seguramente está asociado, por un lado, con el incremento relativo de la PEA del sector terciario, o sea con un proceso de terciarización, y, por el otro, con un aumento del subempleo $\mathrm{y}$ el desempleo urbanos.

\section{LAS CIUdADES SEgÚN SU POBI_ACIÓN ECONÓMICAMENTE aCTIVA AGRÍCOLA}

Dentro de la clasificación funcional de ciudades no se consideró a la PEA agrícola por las razones expuestas en el apéndice. No obstante, dado que existen ciudades con porcientos significativos de PEA agrícola, en este apartado se analiza su situación para investigar si poseen peculiaridades que las distingan.

Tipo de ciudad y PEA agricola. Los porcientos de PEA agrícola en las ciudades consideradas son muy variables. Fluctuaron entre el $28.8 \%$ en Reynosa y el $1.8 \%$ en el Distrito Federal, en 1940; el $17.3 \%$ en Celaya y el $1.3 \%$ en el Distrito Federal, en 1950 , y, en 1960 , entre el $18.1 \%$ en Tepic y el $1.1 \%$ en Tijuana (cuadros A1, A2 y A3).

Como se ve en el cuadro 13 , el número de ciudades con 10.0 a $14.9 \%$, y de $15.0 \%$ y más de PEA agrícola disminuyó notablemente de 1940 a 1960. Esto no quiere decir necesariamente que la PEA agrícola sea cada vez menos significativa en las ciudades estudiadas, sino que considerando que la población de las 37 ciudades se incrementó en ese período, la importancia relativa de la agricultura disminuyó con el aumento en el tamaño de las ciudades, y con el desarrollo del país.

\section{Cuadro 13}

MÉXICO: NúMERO dE CIUdAdES SEGÚN EL PORCIENTO DE POBLACIÓN ECONÓMICAMENTE ACTIVA AGRÍCOLA, 1940-1960

\begin{tabular}{lrrr}
\hline \multirow{2}{*}{$\begin{array}{l}\text { \% de PEA } \\
\text { agricola }\end{array}$} & \multicolumn{3}{c}{ Núroro de ciudades } \\
\cline { 2 - 4 } & 1940 & 1950 & 1960 \\
\hline Total & 37 & 37 & 37 \\
15.0 y más & 8 & 3 & 1 \\
De 10.0 a 14.9 & 10 & 4 & 4 \\
De 5.0 a 9.9 & 10 & 18 & 19 \\
Menos de 5.0 & 9 & 12 & 13 \\
\hline
\end{tabular}

Fuentes: cuadros A1, A2 y A3.

Se esperaría que las ciudades localizadas en zonas agrícolas, con elevados porcientos de PEA agrícola ( $10.0 \%$ y más), se especializaran en servicios, comercio y transportes, actividades dirigidas a satisfacer las necesidades del sector agropecuario. Efectivamente, de las 18 ciudades que en 1940 tenían más de $10.0 \%$ de su PEA en la agricultura, 7 estaban especializadas en servicios, 4 en comercio, 4 en transportes, 2 en la industria de transformación y una en construcción. En 1950 
y 1960 las ciudades de este tipo fueron únicamente comerciales o de servicios, con las excepciones de Irapuato en 1960 y Querétaro en 1950 , que se clasificaron como ciudades industriales. 19

El hecho de que existan dos ciudades indústriales entre las que poseen altos porcientos de PEA agrícola invita a investigar un poco más a fondo su tipo de industrialización. Con este fin se comparó la participación de las principales ramas industriales de las dos ciudades

\section{Cuadro 14}

Monterrey, Irapuato y Querétaro: Participactón dE LAS PRINCIPALES ramas INDUSTRIALES EN LA PRODUCCIÓN BRUTA INDUSTRIAL TOTAL, 1965

(Porcientos)

\begin{tabular}{|c|c|c|c|c|}
\hline \multicolumn{2}{|c|}{$\begin{array}{l}\text { Ramas } \\
\text { industriales }\end{array}$} & \multirow{2}{*}{$\frac{\text { Monterrey }}{8.7}$} & \multirow{2}{*}{ Irapuato } & \multirow{2}{*}{ Queretaro } \\
\hline 20 & $\begin{array}{l}\text { Manufactura de productos ali- } \\
\text { menticios excepto bebidas }\end{array}$ & & & \\
\hline 21 y 22 & $\begin{array}{l}\text { Fabricación de bebidas } y \text { produe } \\
\text { tos del tabaco }\end{array}$ & $5.3^{\mathrm{b} /}$ & 48.2 & 0.0 \\
\hline 34 & Industrialos metâlicas básicas & 21.1 & 0.0 & 0.0 \\
\hline 33 & $\begin{array}{l}\text { Fabricación de otros productos mi } \\
\text { nerales no metálicos }\end{array}$ & 13.0 & 2.7 & 0.4 \\
\hline
\end{tabular}

Fuente: VII Censo Industrial, 1966. Principales características por entidad federativa, municipio y grupo de actividad, SIC. México, 1969.

a Incluye la fabricación de bebidas.

b Excluye la fabricación de tabaco.

en cuestión (Irapuato y Querétaro) con las de Monterrey, ciudad típicamente industrial. En el cuadro 14 se observa que en Monterrey las industrias metálicas básicas y la fabricación de productos minerales no metálicos son las más importantes, mientras que en Irapuato y Querétaro lo son la manufactura de productos alimenticios, de bebidas y de productos del tabaco, cuyos principales insumos provienen dél sector agrícola. Esto explica, en parte, el alto porciento de PEA agrícola en tales ciudades industriales y constituye un ejemplo de desarrollo en dos regiones agrícolas en las cuales se ha fomentado la industrialización estrechamente relacionada con la producción agrícola.

Tamaño de ciudades y PEA agrícola. En un trabajo previo de la investigación sobre la urbanización en México ${ }^{20}$ se planteó la hipótesis, difícil de manejar cuantitativamente en aquella etapa de la investigación, de que las ciudades de menor tamaño deben su impulso de crecimiento a su condición de centros agrícolas y a las actividades comerciales y de servicios relacionadas con esa actividad. Aunque no se puede saber aún en qué medida el comercio y los servicios en estas ciudades son determinados por el sector agrícola, sí se puede aclarar más la hipótesis, observando la relación existente entre el tamaño

19 Las ciudades con $10.0 \%$ y más de PEA agrícola se pueden ver en los cuadros A1, A2 y A3 y su tipo de especialización en el cuadro 1.

20 Unikel y Torres, loc. cit., p. 28. 
de las ciudades y su PEA agrícola con respecto a sus funciones predominantes.

En el cuadro 15 se observa que la mayoría de las ciudades de 100000 y más habitantes se encuentra en los grupos de ciudades con menor porciento de PEA agrícola, o sea que el grupo de ciudades con mayor PEA agrícola son fundamentalmente ciudades pequeñas. Ya se vio que las ciudades de menor tamaño tienden a especializarse en las ramas de comercio y servicios; puede agregarse también que tienen los mayores porcientos de PEA agrícola.

Lonsiderando la PEA agrícola como indicador de la importancia de dicho sector económico en la estructura productiva de una ciudad, se puede decir que en las ciudades con mayor porciento de PEA agrícola - las menores de las 37 ciudades estudiadas- las actividades comerciales y de servicios se orientan en mayor medida a satisfacer los requerimientos del sector agropecuario. Se concluye que, a pesar de que la agricultura no es directamente una actividad predominante en ninguna de las, ciudades estudiadas, en las pequeñas posee mayor importancia relativa; además, constituye un importante mercado para el comercio y los servicios de tales centros urbanos.]

Cuadro 15

México: Crudades según el tamaño de su población y la PEA EN AGRICULTURA, 1940-1960

\begin{tabular}{|c|c|c|c|c|c|}
\hline \multirow{2}{*}{$\begin{array}{c}\text { Manar̃os de } \\
\text { poblacion } \\
\text { (hasitantes) }\end{array}$} & \multirow[b]{2}{*}{ Total } & \multicolumn{4}{|c|}{ PEA aEricola } \\
\hline & & 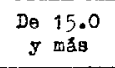 & $\begin{array}{l}\text { De } 10.0 \\
\text { a } 14.9\end{array}$ & $\begin{array}{l}\text { Do } 5.0 \\
\text { a } 9.9\end{array}$ & $\begin{array}{c}\text { Henos de } \\
5.0\end{array}$ \\
\hline \multicolumn{6}{|c|}{1240} \\
\hline Tot23 & 37 & 8 & 10 & 10 & $?$ \\
\hline $\begin{array}{l}\text { Más de } 100 \text { 000 } \\
\text { De } 50 \text { o00 a } 100000 \\
\text { De } 25000 \text { a } 49999 \\
\text { De } 24 \quad 999 \text { y menos }\end{array}$ & $\begin{array}{r}6 \\
8 \\
12 \\
11\end{array}$ & $\begin{array}{l}0 \\
0 \\
3 \\
5\end{array}$ & $\begin{array}{l}1 \\
0 \\
6 \\
3\end{array}$ & $\begin{array}{l}0 \\
5 \\
2 \\
3\end{array}$ & $\begin{array}{l}5 \\
3 \\
\vdots \\
0\end{array}$ \\
\hline \multicolumn{6}{|c|}{1950} \\
\hline Total & 37 & 3 & 4 & 13 & 12 \\
\hline $\begin{array}{l}\text { Mas de } 100 \text { o00 } \\
\text { Da } 50 \quad 000 \text { a } 100 \quad 000 \\
\text { De } 25000 \text { a } 49999 \\
\text { De } 24 \quad 999 \text { y menos }\end{array}$ & $\begin{array}{r}11 \\
12 \\
13 \\
1\end{array}$ & $\begin{array}{l}0 \\
1 \\
2 \\
0\end{array}$ & $\begin{array}{l}1 \\
0 \\
3 \\
0\end{array}$ & $\begin{array}{l}3 \\
7 \\
7 \\
1 \\
1\end{array}$ & $\begin{array}{l}7 \\
4 \\
1 \\
0\end{array}$ \\
\hline \multicolumn{6}{|c|}{1960} \\
\hline Total & 37 & 3 & 4 & 19 & 13 \\
\hline $\begin{array}{l}\text { Nas de } 100000 \\
\text { De } 50000 \text { a } 100000\end{array}$ & $\begin{array}{l}17 \\
20\end{array}$ & $\begin{array}{l}0 \\
1\end{array}$ & $\begin{array}{l}1 \\
3\end{array}$ & $\begin{array}{r}7 \\
12\end{array}$ & $\begin{array}{l}9 \\
4\end{array}$ \\
\hline
\end{tabular}

Fuentes: cuadros 1, A1, A2 y A3.

a Porciento de PEA agrícola respecto de la total.

Tasa de crecimiento y PEA agrícola. Previamente se determinó que no todas las ciudades de servicios observan un rápido crecimiento, pero que la mayoría de las ciudades de alta tasa de crecimiento soñ 
de servicios. Dado que las ciudades que tienen altos porcientos de PEA agrícola están especializadas en comercio y servicios, resultaría lógico esperar que entre ellas hubiera ciudades de crecimiento elevado. Efectivamente, de las 9 que en el decenio 1940-1950 tuvieron una tasa de crecimiento de $7.5 \%$ o más, 6 eran ciudades con elevado porciento de PEA agrícola ( $10.0 \%$ o más).

De 1940 a 1950 la agricultura nacional creció a una tasa de $7.5 \%$ anual, contra el $6.0 \%$ del producto total, por lo que se puede pensar que la dinámica de la agricultura fue uno de los elementos que hicieron posible el alto crecimiento alcanzado por estas ciudades. De 1950 a 1960 la tasa de crecimiento de la agricultura descendió a $4.3 \%$, y sólo dos de las 6 ciudades con alto porciento de PEA agrícola (que en 1940-1950 habían tenido una tasa rápida de crecimiento: Ciudad Juárez, Matamoros, Mexicali y Ciudad Obregón, Reynosa y Culiacán), Ciudad Obregón y Mexicali, mantuvieron un crecimiento rápido. Lo anterior indica que el elevado crecimiento agrícola de 1940 a 1950, aunado al desarrollo del comercio y los servicios que éste originó, fue uno de los factores que fomentaron el acelerado crecimiento de las ciudades estabtecidas en las zonas agrícolas, el cual se frenó al descender fuertemente la tasa de crecimiento del producto agrícola en el período 1950-1960.

Todo lo anterior parece señalar que el descenso significativo en la tasa de crecimiento de las principales ciudades de la frontera norte, de 1960 a 1970, se debió a una disminución adicional del desarrollo agrícola. Los servicios y el comercio, ligados al turismo en dichas ciudades, al parecer no fueron suficientemente dinámicos para mantener las elevadas tasas de crecimiento de la población registrada de 1940 a 1960 .

\section{ESPECIALIZACIÓN DE LAS CIUDADES SEGÚN REGIONES ECONÓMICAS}

Las 37 ciudades analizadas absorben entre el 60 y el $70 \%$ de la PEA de los sectores secundario y terciario del país. Esto explica su indiscutible importancia en el desarrollo económico de México. En esta sección se trata de ver si la anterior afirmación es también válida a nivel regional; además se pretende determinar que existe un patrón definido entre el número y el tipo de ciudades establecidas en las zonas del país según diferente nivel de desarrollo económico en 1960,21 durante el período 1940-1960 (ver mapa 1). Las preguntas a contestar son: ¿cuál es la primera función predominante de las ciudades localizadas en las

$21[$ Se utilizó una zonificación del país compuesta por cuatro zonas de diferentes niveles de desarrollo económico, elaborada con base en la relación entre el producto bruto interno y la población económicamente activa (PBI/PEA) de las entidades federativas que la forman. Para 1960 esta zonificación está constituida por: Zona I (la más desarrollada) integrada por el Distrito Federal, Nuevo León, Baja California Norte, Sonora, Chihuahua y Coahuila; Zona II (de desarrollo medio), Sinaloa, Veracruz, Baja California Sur, Tamaulipas y Tabasco; Zona III (de bajo desarrollo), formada por Yucatán, México, Campeche, Morelos, Jalisco, Colima, Durango, Nayarit, Guanajuato, Quintana Roo, Aguascalientes y San Luis Potosí (de muy bajo desarrollo), formada por Guerrero, Querétaro, Hidalgo, Zacatecas, Puebla, Chiapas, Michoacán, Tlaxcala y Oaxaca. Las zonificaciones económicas para 1940 y 1950 no se utilizaron por las complicaciones que esto introducía al análisis. Véase L. Unikel, G. Garza y A. González, loc. cit. 


\section{Mapa 1}

MÉXico: TIPO Y NÚMERO DE FUNCIONES PREDOMINANTES DE LAS PRINCIPALES CIUDADES, 1940-1960

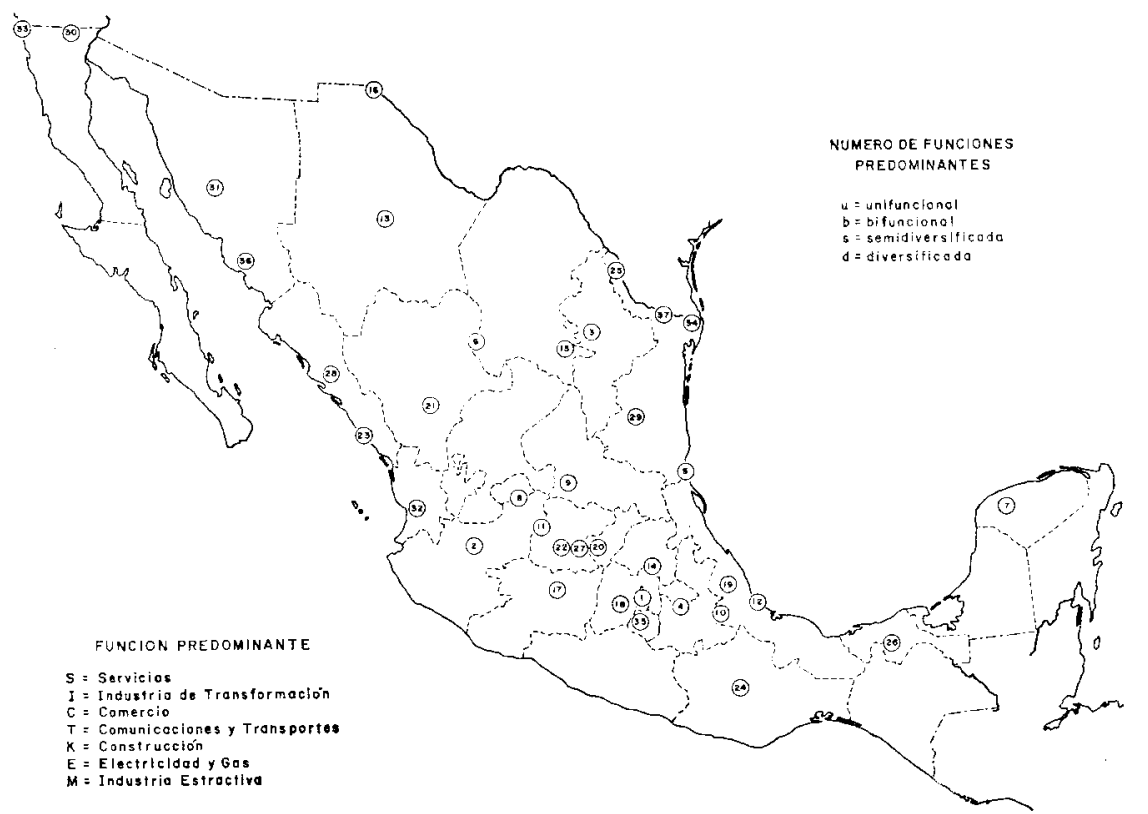

Nota: Como ejemplo explicativo se presenta el siguiente caso: 3, Monterrey, N. L. III (dsb). El 3 corresponde a la localización de la ciudad; N. L. a la entidad federativa; las I indican la primera función predominante en 1940,1950 y 1960, respectivamente; (dsb) son el número de funciones predominantes en 1940,1950 y 1960 , respectivamente.

\begin{tabular}{|c|c|c|c|c|c|c|c|c|}
\hline \multirow{2}{*}{$\begin{array}{c}\begin{array}{c}\text { Núm. } \\
\text { de } \\
\text { orden }\end{array} \\
1\end{array}$} & Ciudad & $\begin{array}{c}\text { Entidad } \\
\text { federativa }\end{array}$ & \multicolumn{3}{|c|}{$\begin{array}{c}\text { Primera función } \\
\text { predominante }\end{array}$} & \multicolumn{3}{|c|}{$\begin{array}{c}\text { Número } \\
\text { de funciones } \\
\text { predominantes }\end{array}$} \\
\hline & Cd. de México & D. F. & $\mathrm{S}$ & $\mathrm{S}$ & $\mathrm{S}$ & $\mathbf{u}$ & $b$ & $S$ \\
\hline 2 & Guadalajara & JAL. & I & I & $\bar{I}$ & $s$ & d & $\$$ \\
\hline 3 & Monterrey & N. L. & $\bar{I}$ & $\bar{I}$ & $\mathrm{I}$ & d & s & $\mathrm{b}$ \\
\hline 4 & Puebla & PUE. & I & $\mathrm{I}$ & $\mathrm{I}$ & s & b & $s$ \\
\hline 5 & Tampico & TAMPS. & M & $\mathrm{M}$ & I & $\mathrm{s}$ & s & $\mathrm{s}$ \\
\hline 6 & Torreón & COAH. & $\mathrm{T}$ & C & $\bar{T}$ & d & $\mathrm{d}$ & $\mathrm{s}$ \\
\hline 7 & Mérida & YUC. & $\mathrm{C}$ & $\mathrm{C}$ & $\mathrm{C}$ & $\mathrm{d}$ & $\mathrm{s}$ & $\mathrm{s}$ \\
\hline 8 & Aguascalientes & $\mathrm{AGS}$ & $\mathrm{T}$ & $T$ & I & b & $s$ & $\mathrm{~s}$ \\
\hline 9 & San Luis Potosí & S. L. P. & $\mathrm{T}$ & $\mathrm{T}$ & $\mathrm{T}$ & $s$ & $s$ & $s$ \\
\hline 10 & Orizaba & VER. & $\mathrm{I}$ & I & I & $\mathbf{S}$ & $b$ & $\mathrm{~s}$ \\
\hline 11 & León & GTO. & $\bar{I}$ & $\bar{I}$ & $\bar{x}$ & b & $\mathbf{u}$ & $\mathrm{u}$ \\
\hline 12 & Veracruz & VER. & $\mathrm{T}$ & $\mathrm{T}$ & $\mathrm{T}$ & d & $\mathrm{d}$ & $\mathrm{d}$ \\
\hline 13 & Chihuahua & CHIH. & $\mathrm{T}$ & $\mathrm{T}$ & $\mathrm{K}$ & $\mathrm{d}$ & $\vec{b}$ & $\mathrm{~d}$ \\
\hline 14 & Pachuca & $\mathrm{HGO}$ & $\mathrm{M}$ & $\mathrm{M}$ & $\mathrm{M}$ & b & $\mathrm{s}$ & $\mathrm{s}$ \\
\hline 15 & Saltillo & COAH. & $\mathrm{T}$ & $\mathrm{T}$ & $\mathrm{K}$ & $s$ & $\mathrm{~s}$ & $\mathrm{~d}$ \\
\hline 16 & Cd. Juárez & CHIH. & $\mathrm{C}$ & $\mathrm{K}$ & $\mathrm{S}$ & d & $\mathrm{d}$ & $\mathrm{s}$ \\
\hline 17 & Morelia & MICH. & $S$ & $\mathrm{~S}$ & $S$ & $\mathrm{~s}$ & $\mathrm{~s}$ & $\mathrm{~s}$ \\
\hline
\end{tabular}

(continúa) 
(continuación)

\begin{tabular}{|c|c|c|c|c|c|c|c|c|}
\hline \multirow{2}{*}{$\begin{array}{c}\begin{array}{c}\text { Nimn. } \\
\text { de } \\
\text { orden }\end{array} \\
18\end{array}$} & \multirow[t]{2}{*}{ Ciudad } & \multirow{2}{*}{$\frac{\begin{array}{c}\text { Entidad } \\
\text { federativa }\end{array}}{\mathrm{MEX} .}$} & \multicolumn{3}{|c|}{$\begin{array}{c}\text { Primera función } \\
\text { predominante }\end{array}$} & \multicolumn{3}{|c|}{$\begin{array}{c}\text { Número } \\
\text { de funciones } \\
\text { predominantes }\end{array}$} \\
\hline & & & $\mathrm{S}$ & $S$ & $\mathrm{~S}$ & $s s$ & $s$ & $\mathrm{~d}$ \\
\hline 19 & Jalada & VER. & $S$ & $S$ & $S$ & $\mathrm{~d} s$ & 5 & $\mathrm{~s}$ \\
\hline 20 & Querétaro & QRO. & I & I & $\mathrm{C}$ & d c & d & $\mathrm{s}$ \\
\hline 21 & Durango & DGO. & $\mathrm{C}$ & $\mathrm{S}$ & $S$ & d c & d & $\mathrm{S}$ \\
\hline 22 & Irapuato & GTO. & $I$ & $\mathrm{C}$ & I & d c & d & \\
\hline 23 & Mazatlán & SIN. & $\mathrm{T}$ & $\mathrm{T}$ & $\mathrm{T}$ & $\mathrm{s}$ & $\mathrm{b}$ & \\
\hline 24 & Oaxaca & OAX. & I & $\hat{S}$ & $\mathrm{~S}$ & b $c$ & u & \\
\hline 25 & Nuevo Laredo & TAMPS. & $\mathrm{T}$ & $\mathrm{T}$ & $\mathrm{S}$ & d d & d & $\mathrm{d}$ \\
\hline 26 & Villahermosa & TAB. & $\mathrm{T}$ & $\mathrm{S}$ & C & d d & d & $d$ \\
\hline 27 & Celaya & GTO. & $\mathrm{C}$ & $\mathrm{C}$ & $\mathrm{C}$ & d 1 & $\mathrm{~b}$ & $\mathrm{~s}$ \\
\hline 28 & Culiacán & SIN. & $S$ & $\mathrm{C}$ & $\mathrm{C}$ & b c & d & $\mathrm{d}$ \\
\hline 29 & Cd. Victoria & TAMPS. & $\mathrm{S}$ & $\mathrm{S}$ & $\mathrm{S}$ & $\mathrm{d}$ & d & $\mathrm{d}$ \\
\hline 30 & Mexicali & B. C. N. & $\mathrm{S}$ & $S$ & $S$ & $\mathbf{u}$ & $\mathrm{s}$ & $\mathrm{d}$ \\
\hline 31 & Hermosillo & SON. & $\mathrm{S}$ & $S$ & $\mathrm{~S}$ & s c & d & $\mathrm{d}$ \\
\hline 32 & Tepic & NAY. & $S$ & $\mathrm{~S}$ & $\mathrm{~K}$ & s $\mathrm{b}$ & $\mathrm{b}$ & $\mathrm{s}$ \\
\hline 33 & Tijuana & B. C. N. & $\mathrm{S}$ & $\mathrm{S}$ & $\mathrm{S}$ & $\mathrm{s} c$ & d & $\mathrm{C}^{\mathrm{i}}$ \\
\hline 34 & Matamoros & TAMPS. & $\mathrm{C}$ & $\mathrm{C}$ & $\mathrm{C}$ & $b s$ & $\mathrm{~s}$ & $s$ \\
\hline 35 & Cuernavaca & MOR. & $\mathrm{S}$ & $\mathrm{S}$ & $\mathrm{S}$ & $s s$ & $s$ & \\
\hline 36 & Cd. Obregón & SON. & $\mathrm{K}$ & $\mathrm{K}$ & C & s s & $\mathrm{s}$ & $\mathrm{d}$ \\
\hline 37 & Reynosa & TAMPS. & $\mathrm{S}$ & $\mathrm{C}$ & $\mathrm{C}$ & d c & $\mathrm{d}$ & $\mathrm{d}$ \\
\hline
\end{tabular}

diversas zonas de desarrollo económico? $\mathrm{y}$, esas ciudades $\dot{c}$ son unifuncionales, bifuncionales, semidiversificadas o diversificadas?

En cuanto al número de ciudades en cada una de las cuatro zonas se puede afirmar que no hay relación directa entre éste y el nivel de desarrollo. Así, las zonas más desarrolladas no necesariamente deben poseer un mayor número de ciudades importantes. Sin embargo, las zonas de mayor desarrollo (I y II) poseen 1.91 ciudades por entidad, mientras que las zonas de bajo y muy bajo desarrollo tuvieron 0.76 ciudades por entidad (cuadro 16). Más aún, las 10 ciudades de la zona I contaban con el $73.3 \%$ de la PEA total de las entidades que la conforman, mientras que las 11 ciudades para cada una de las zonas II y III, sólo tenían el 18.5 y el $19.6 \%$, respectivamente; y las 5 de la zona IV, únicamente el $6.0 \%$. Esto ratifica, en términos generales, que el número de ciudades importantes no necesariamente se relaciona con el desarrollo económico; éste se asocia más bien con otras características

\section{Cuadro 16}

MÉXICO: NÚMERo DE CIUdADES SEgúN ZONAS DE DIFERENTE NIVEL DE DESARROLLO ECONÓMICO, 1960

\begin{tabular}{|c|c|c|c|c|}
\hline \multirow{2}{*}{$\begin{array}{l}\text { Zonas segtin dega } \\
\text { rroll lo eaonómico }\end{array}$} & \multicolumn{2}{|c|}{ Nómero de } & \multirow{2}{*}{$\frac{\text { cindades }}{\text { ontidades }}$} & PEA ciudadeg \\
\hline & ciadades & entidadea & & PEA entidades \\
\hline Total & 37 & 32 & 1.15 & 30.6 \\
\hline $\begin{array}{ll}\text { Zona I } \\
\text { Zona II } \\
\text { Zona III } \\
\text { Zona IV }\end{array}$ & $\begin{array}{r}10 \\
11 \\
11 \\
5\end{array}$ & $\begin{array}{r}16 \\
5 \\
12 \\
9\end{array}$ & $\begin{array}{l}1.66 \\
2.20 \\
0.91 \\
0.55\end{array}$ & $\begin{array}{r}73.3 \\
18.5 \\
19.6 \\
6.0\end{array}$ \\
\hline
\end{tabular}

Fuentes: Mapa 1, y L. Unikel, G. Garza y A. González, loc. cit.

a Las cifras son cocientes del número de ciudades por entidad y de la PEA de las ciudades entre la de las entidades de cada zona. 
de la urbanización. Tal es el caso de la participación de la estructura económica de las ciudades con respecto a la de su región. Así, un estado como Guanajuato, de la zona II (de bajo desarrollo), tiene tres ciudades, mientras que Nuevo León, de la zona más desarrollada, sólo posee una, y Sonora, también de la zona I, tiene dos. A nivel internacional sucede algo semejante: países subdesarrollados, como la India, tienen más ciudades que países desarrollados como Francia. En este caso, como en el de México, es indiscutible que factores tales como el tamaño territorial y de la población del país y de las ciudades, el producto que generan, su estructura ocupacional, su localización geográfica, etc., influyen más en el desarrollo económico que el simple número de ciudades. Sin embargo, sí se puede decir que la escasez de ciudades principales, como acontece en la zona IV se relaciona con un bajo nivel de desarrollo económico.

Lo anterior se complementa al observar que las ciudades de la zona más desarrollada absorben el $73.3 \%$ de la PEA total de las entidades que la forman y el $91.0 \%$ de la PEA no agrícola, mientras que un mayor número de ciudades en la zona II (de desarrollo medio) poseen sólo el 18.5 y el $45.7 \%$ de la PEA, respectivamente. La PEA no agrícola restante se encuentra dispersa en ciudades menores y en localidades no urbanas.

De aquí se deriva que, para fines de planeación del desarrollo económico, sea importante tomar en consideración las interacciones del sector urbano con el rural, pues se considera conveniente que un porciento significativo de la producción no agrícola regional descanse en el primero. Éste posee factores positivos para el desarrollo tales como: economías externas y de escala, infraestructura y servicios, diversificación de la mano de obra, mercado para los productos, etc., que son elementos necesarios, aunque no suficientes, para el crecimiento de los sectores secundario y terciario. A pesar de la aceptación con que cuenta la teoría de los polos de desarrollo y de que una distribución más uniforme de la población urbana es favorable al desarrollo económico, a la fecha no existe un consenso en cuanto a cuál debe ser la distribución óptima de la población urbana y rural que conduzca a un mayor desarrollo económico.

En cuanto al tipo de especialización de las ciudades, según las cuatro zonas, se encontró que para 1940,1950 y 1960 , las de servicios son las más importantes en la zona más desarrollada. Le siguen en importancia las especializadas en transporte, en 1940, y en construcción en 1950 y 1960. El hecho de que las ciudades de estos tipos sean las más importantes en la zona I, y no las industriales, como podría esperarse, se puede interpretar, entre otras razones, como un reflejo de las relaciones de intercambio comercial y de servicios con los Estados Unidos. El impacto de la economía de ese país sobre la zona I se refleja en las transacciones fronterizas, el contrabando, el turismo, la agricultura y la ganadería de exportación, etc., sobre la mayoría de las ciudades de la frontera.

La zona de desarrollo medio (II) está compuesta por 5 entidades costeras, por lo que la existencia de ciudades en dichas entidades justifica el que en 1940 se especializaran principalmente en transportes y servicios. Sin embargo, en 1950 y 1960 las que predominaron en 
transportes disminuyeron notablemente; por el contrario, las comerciales, junto con las de servicios, fueron mayoritarias.

Las entidades del centro de la República, algunas del este, así como las de la península de Yucatán, conforman la zona de bajo desarrollo. Sus ciudades, en 1940, predominaron en servicios, pero también fueron importantes las comerciales e industriales. En 1950 predominaron las dos primeras, mientras que en 1960 las industriales fueron las más significativas. Esta evolución señala que aunque se trata de una zona de bajo desarrollo, el sector industrial de sus ciudades principales mostró un incrémento relativamente más rápido de la PEA industrial de 1950 a 1960. Este hecho puede ser un indicio que merece un estudio más profundo de esta zona para un posible programa de fomento industrial en sus principales ciudades, dada la posibilidad de emplear la mano de obra industrial, la estructura urbana con características industriales $\mathrm{y}$, lo que es fundamental, la cercanía de algunas de sus entidades a la zona metropolitana de la ciudad de México.

Finalmente, la zona de muy bajo desarrollo se compone principalmente de entidades localizadas en el sur y el sureste del país y algunas entidades del centro. Dado que en esta región sólo existen 5 ciudades es difícil hacer generalizaciones. En los tres años estudiados dos ciudades eran de servicios (Morelia y Oaxaca); dos industriales en 1940 (Querétaro y Puebla), y una en 1950 y 1960 (Querétaro y Puebla, respectivamente); en consecuencia se puede considerar este tipo de ciudades como las predominantes en la zona. No obstante ser la zona menos desarrollada, algunas de sus entidades y ciudades tienen características favorables para el desarrollo industrial, pues cuenta con algunos de los factores de localización señalados para la zona III. Este sería el caso de Querétaro y Puebla.

\section{CONCLUSIONES}

1. La baja participación del sector industrial y la elevada del sector primario y terciario dentro de la estructura ocupacional en México se refleja en el predominio de las ciudades que se especializan principalmente en servicios, comercio y transportes. Estas constituyen, de 1940 a 1970 , alrededor del $70 \%$ del total; el $30 \%$ restante se especializó en industria de transformación $(20 \%)$, y construcción y minería $(10 \%)$.

2. No obstante, se observa fuerte tendencia hacia la diversificación de funciones predominantes en las principales ciudades. Esto implica, necesariamente, aumento de la participación de las ciudades en la PEA no agrícola del país, adquiriendo éstas cada vez más importancia en la producción global de los bienes no primarios.

3. Del análisis de los tipos de especialización de las ciudades según sus tamaños de población, tasas de crecimiento, localización geográfica y grado de concentración interna de su estructura ocupacional (IED), se obtuvo la siguiente tipología de ciudades:

a) Las ciudades especializadas en servicios son de tamaño pequeño o mediano y generalmente capitales o fronterizas. Su población crece a ritmos alto y medio y ocupan el segundo lugar en cuanto al grado de concentración de su PEA. 
b) Las ciudades industriales son de tamaño medio o grande, se localizan en el interior de la República, algunas de ellas son capitales y su tasa de crecimiento es media. Son las que manifiestan mayor grado de concentración en su estructura ocupacional.

c) Las ciudades que se especializan en transporte son de tamaño medio, portuarias y de bajo crecimiento y mayor diversificación en su PEA.

d) Las que se especializan en comercio son pequeñas y sus tasas de crecimiento altas o medias. Se encuentran en la frontera o en el interior y manifiestan una diversificación intermedia en su estructura ocupacional.

e) Las pocas ciudades especializadas en industria de la construcción son pequeñas, de crecimiento alto, concentración económica media, y no mostraron ningún patrón en cuanto a su localización geográfica.

f) Las especializadas en minería fueron las de más bajo crecimiento y PEA más diversificada.

4. El aumento de las ciudades especializadas en servicios, de 1960 a 1970, refleja fuerte crecimiento en la PEA de esta rama. Esto puede significar que se ha acentuado el proceso de terciarización en las principales ciudades del país, siguiendo la tendencia originada de 1950 a 1960.

5. La gran dinámica del sector agrícola en el período 1940-1950 fomentó un crecimiento rápido de población de las ciudades establecidas en las zonas de agricultura moderna. Éste se frenó al disminuir fuertemente la dinámica del producto agrícola en el período 1950-1960.

6. No existe relación directa entre el desarrollo económico de las regiones y su número de ciudades importantes. Sin embargo, la participación de los centros urbanos en la estructura ocupacional (PEA) de las regiones es más alta al aumentar el nivel de desarrollo. Podría decirse que la existencia de ciudades importantes es condición necesaria pero no suficiente del desarrollo.

7. Las ciudades de la zona más desarrollada -el Distrito Federal y los estados fronterizos del norte, excepto Tamaulipas- son típicamente de servicios y reflejan la importancia del impacto comercial de Estados Unidos.

8. Las ciudades industriales se encuentran, contra lo que se esperaba, de manera principal, en la zona de bajo desarrollo económico.

9. Los programas de desarrollo regional deberían evaluar la viabilidad de aprovechar la infraestructura industrial de las ciudades de la zona de bajo desarrollo.

\section{APÉNDICE METODOLÓGICO}

\section{A. El ÍNDICE DE tRABAJADORES EXCEJENTES}

Para clasificar las principales ciudades de México se ha seleccionado como instrumento el índice de trabajadores excedentes. ${ }^{1}$

1 Éste y el cociente de localización o índice de especialización local han sido aplicados, principalmente, en la identificación general de las "actividades básicas" 
El índice mide el número absoluto de trabajadores excedentes, a través de la diferencia entre el empleo de las ramas de actividad de la ciudad y su participación en el empleo nacional de dichas ramas. Su expresión numérica es:

$$
S=\frac{e_{i}-e_{t}}{E_{t}} \cdot E_{i}
$$

donde $S$ es el número absoluto de trabajadores excedentes en la rama de actividad $i ; e_{i}$ y $E_{i}$ son la PEA local y nacional en la actividad $i ; e_{t}$ y $E_{t}$ son la PEA total local y nacional, respectivamente. Esta forma es apropiada únicamente para comparaciones intra-ciudad, pero convertida en términos relativos permite realizar comparaciones intra e inter-ciudades. Esto último se logra dividiendo el índice en su forma absoluta entre la PEA total de la ciudad, $e_{t}$, o entre el total de sus trabajadores excedentes:

$$
e_{i}-\frac{e_{t}}{E_{t}} \cdot E_{i}
$$

Se optó por utilizar la primera forma relativa, por la sencillez de cálculo y, además, porque mediante ambas expresiones se llega a rangos idénticos en los valores de los trabajadores excedentes en las distintas ramas de actividad para las ciudades estudiadas. ${ }^{2}$ La expresión relativa finalmente aplicada en este estudio es la siguiente:

$$
\frac{e_{i}-\frac{e_{t}}{E_{t}} \cdot E_{i}}{e_{f}}=\frac{e_{i}}{e_{t}}-\frac{E_{i}}{E_{t}}
$$

La característica principal de este índice es que señala la importancia de cada actividad económica para la ciudad, considerando la magnitud de la rama de actividad del país.

En vista de las limitaciones del índice para determinar la base económica de una ciudad,; algunos investigadores lo han utilizado con fines menos ambiciosos, más descriptivos que explicativos, como elementos que facilitan el entendimiento de la estructura económica de una ciudad o región y de sus relaciones con otras. No obstante lo anterior, el índice de trabajadores excedentes también presenta limitaciones como herramienta para la clasificación de ciudades. La principal estriba en el hecho de que los resultados pueden cambiar dependiendo de la variable contra la cual se comparan los datos locales; en el caso de la fórmula anterior, la $E$.

Siendo el propósito central de la presente clasificación el señalar el tipo y grado de especialización de las principales ciudades, dentro de la estructura económica nacional según la PEA por ramas de actividad, se optó por

de una ciudad, las cuales - según una teoría de la economía urbana- constituyen la "base económica de la localidad". La literatura sobre este tema es aún más amplia que la correspondiente a la clasificación de ciudades, por lo que sólo se citan dos trabajos importantes donde se emplea el índice de trabajadores excedentes para determinar la base económica: Mattila y Thompson, op. cit., y P. D. McGovern, "Identifying Exporting Industries", Journal of the American Institute of Planners, Vol. 27, Núm. 2, mayo de 1961, pp. 144-150.

2 Mattila y Thompson, op. cit., pp. 337-342.

3 McGovern, loc. cit y W. Isard, Methods of Regional Analysis, Cambridge, Mass., MIT Press, 1969, pp. 189-205. 
utilizar como marco de referencia la PEA total de las diversas ramas del país.

La rama agrícola no se consideró en la clasificación de las ciudades: En primer lugar, porque la actividad agrícola no se realiza en el ámbito físico de la ciudad (aunque parte de la PEA agrícola habite en las ciudades). En segundo y en parte debido a lo anterior, porque siendo México un país predominantemente agrícola, ninguna ciudad se clasificaría como tal al compararla con el total nacional: $65.4 \%$ en 1940, $58.3 \%$ en 1950 y $54.1 \%$ en 1960.

Con miras a evaluar el grado de variabilidad de la aplicación del índice, modificando el marco de referencia $E$, se analizaron dos alternativas adicionales: a) con la estructura promedio de la PEA de las 37 ciudades; $b$ ) con la estructura promedio de la PEA de las 36 ciudades, excluyendo a la ciudad de México debido al gran peso que tiene en el conjunto urbano estudiado.

Los resultados de las tres clasificaciones muestran grandes semejanzas y, comparando la clasificación con base en el total nacional para $1960 \mathrm{con}$ las obtenidas para el total de las 37 ciudades, así como para el de las 36 , se observa que sólo existen 5 y 8 ciudades, respectivamente, que presentan cambios en su primera función predominante.

La semejanza de las 3 clasificaciones se explica por la gran importancia que poseen las 37 ciudades dentro de la estructura ocupacional del país. En 1940, las 37 ciudades poseían entre el $50 \%$ y el $60 \%$ de la PEA de las ramas de actividad no agrícola, con excepción de la minería, aumentando su participación en 1950 y en 1960 hasta poseer, en este último año, entre el $60 \%$ y el $70 \%$ de la PEA del país en dichas ramas.

Cada una de las clasificaciones resultantes satisface un propósito particular. Esta aseveración se puede hacer extensiva a toda tipología urbana ya que, de hecho, no se ha llegado a una sola que sea idónea para cualquier uso, dada la arbitrariedad implícita en toda clasificación.

La relativa semejanza de los resultados entre las tres clasificaciones, permite, con las reservas del caso, considerar útil a la primera, tanto para analizar la naturaleza económica general de las principales ciudades dentro de la estructura económica no agrícola nacional, como también para estudiar diferencias interurbanas.

\section{B. El INNDICE DE DIFERENCIACIÓN ECONÓMICA (IED)}

El índice de trabajadores excedentes expresa el tipo de especialización de las ciudades. No obstante, no señala el grado de esa especialización dentro de la estructura ocupacional de la ciudad. Por ejemplo, respecto a dos ciudades clasificadas de servicios, v. gr.: Mexicali y la ciudad de México en 1940, no se sabe en cuál de ellas existe mayor concentración de su estructura ocupacional. Con objeto de cuantificar el grado de concentración de las diferentes ramas de actividad para las 37 ciudades, se utilizó el índice de diferenciación económica (IED) cuya expresión matemática es :

$$
\mathrm{IED}=\sum_{i=1}^{n}\left(\frac{n}{n-1}\right)\left(p_{i}-\frac{1}{n}\right)^{2}
$$

en donde $n$ es el número de ramas de actividad y $P$ es el porciento de PEA para cada rama de actividad $i$. El indice tiene la característica de que varía entre 0 y 1 , expresando en el primer caso una PEA distribuida en forma proporcionalmente uniforme y en el segundo toda la mano de obra concentrada en una sola actividad. 
Como ejemplos de su aplicación se han tomado las ciudades de León, Irapuato, Pachuca y Tampico, ciudades las dos primeras con primera función predominante en industria y las últimas en actividades extractivas. El IED para León e Irapuato en 1960, fue de 0.276 y 0.130 , respectivamente, lo que señala, entre otras características, una diferencia sustancial en su grado de industrialización, siendo que el porciento de PEA industrial en León era de $54.7 \%$ y de $31.3 \%$ en Irapuato. En los casos de Pachuca y Tampico, los IED se acercan a cero: 0.082 en la primera en 1960 y 0.059 en la segunda para 1940 y 1950 ; sin embargo, la especialización funcional es distinta : transportes en Tampico y servicios en Pachuca.

El IED se considera un auxiliar útil de la clasificación funcional, y su análisis, no obstante la brevedad con que se hizo en este trabajo, aportó una idea de su posible aplicación. ${ }^{4}$

4 Para mayores detalles sobre el IED, véase E. C. Amemiya, "Measurement of Economic Differentiation", Journal of Regional Science, Vol. 5, Núm. 1, 1963, pp. 84-85, y Amemiya, "Economic Differentiation...", loc. cit., pp. 57-61. 
Cuadro A 2

MÉxTOO: PEA DE 12 aÑos Y Más POR ÁREAS URBANAS Y RAMAS DE ACTTVDAD, 1950 (Porcientos)

\begin{tabular}{|c|c|c|c|c|c|c|c|c|c|}
\hline \multirow[b]{2}{*}{ Giudadege } & \multirow[b]{2}{*}{$\begin{array}{l}\text { PEA } \\
\text { Total }\end{array}$} & \multicolumn{8}{|c|}{ Ramas de actividad } \\
\hline & & $\begin{array}{l}\text { Agrope } \\
\text { cuariab/ }\end{array}$ & $\begin{array}{l}\text { Extrac } \\
\text { tiva }\end{array}$ & $\begin{array}{l}\text { Triansfor } \\
\text { mación }\end{array}$ & $\begin{array}{c}\text { Construc } \\
\text { ción }\end{array}$ & $\begin{array}{l}\text { Electri } \\
\text { cidad }\end{array}$ & $\begin{array}{l}\text { Comer } \\
\text { cio }\end{array}$ & $\begin{array}{c}\text { Comunica } \\
\text { oiones } y \\
\text { transpor } \\
\text { tes } \\
\end{array}$ & $\begin{array}{l}\text { Servi } \\
\text { oios }\end{array}$ \\
\hline Total & 2098099 & 3.46 & 0.90 & 29.53 & 7.77 & 0.34 & 19.14 & 8.06 & 30.30 \\
\hline Aguascalientes & $27 \quad 143$ & 9.43 & 0.50 & 29.39 & 6.52 & 0.75 & 19.91 & 13.76 & 19.74 \\
\hline Mexicali & 20199 & 17.18 & 0.56 & 18.88 & 6.72 & 0.76 & 20.16 & 5.85 & 29.89 \\
\hline Tijuana & 17313 & 2.77 & 0.07 & 21.49 & 8.89 & 1.40 & 23.18 . & 6.07 & 36.43 \\
\hline Saltillo & 22440 & 7.14 & 0.40 & 28.56 & $9 \cdot 17$ & 0.85 & 17.93 & 10.55 & 25.40 \\
\hline Torreón & 57531 & 11.65 & 1.23 & 26.07 & 8.05 & 0.86 & 20.99 & 9.24 & 21.91 \\
\hline Cd. Juárez & 38565 & 8.88 & 0.56 & 21.81 & 12.68 & 0.83 & 18.79 & 6.90 & 29.55 \\
\hline Chihuahua & $\begin{array}{r}29216 \\
\end{array}$ & 3.41 & 0.83 & 25.52 & 11.79 & 0.77 & 19.20 & 11.71 & 26.77 \\
\hline cd. de México & 1045080 & 1.29 & 0.50 & 30.02 & 7.17 & 0.88 & 18.43 & 7.12 & 34.59 \\
\hline Durango & 18776 & 8.12 & 1.73 & 21.40 & 7.92 & 0.62 & 19.28 & 9.22 & 31.71 \\
\hline Celaya & 10858 & 17.32 & 0.19 & 25.06 & 6.35 & 1.17 & 23.83 & 5.39 & 20.69 \\
\hline Irapuato & 14.782 & 15.83 & 0.09 & 28.49 & $7 \cdot 13$ & 0.69 & $2 \uparrow .44$ & 7.78 & 18.55 \\
\hline León & 38598 & 5.02 & 0.11 & 55.76 & 5.22 & 0.50 & 16.50 & 3.22 & 13.67 \\
\hline Pachuca & 19080 & 1.98 & 15.56 & 21.56 & 6.09 & 1.14 & 18.28 & 5.28 & 29.60 \\
\hline $\begin{array}{l}\text { Guadalajara } \\
\text { Tolvaa }\end{array}$ & $\begin{array}{l}130973 \\
18\end{array}$ & 2.96 & 0.11 & $\begin{array}{l}32.49 \\
277.79\end{array}$ & $9: 59$ & 0.88 & 20.07 & $\begin{array}{l}7.61 \\
8.88\end{array}$ & $\begin{array}{l}26.29 \\
32.52\end{array}$ \\
\hline $\begin{array}{l}\text { Toluoa } \\
\text { Morelia }\end{array}$ & $\begin{array}{l}18718 \\
20282\end{array}$ & $\begin{array}{l}3.35 \\
9.33\end{array}$ & $\begin{array}{l}0.38 \\
0.26\end{array}$ & $\begin{array}{l}27.79 \\
21.63\end{array}$ & $\begin{array}{l}5.09 \\
8.14\end{array}$ & $\begin{array}{l}0.77 \\
0.36\end{array}$ & $\begin{array}{l}21.22 \\
21.72\end{array}$ & 8.88 & $\begin{array}{l}32.52 \\
32.15\end{array}$ \\
\hline $\begin{array}{l}\text { Morelia } \\
\text { Guemavaca }\end{array}$ & $\begin{array}{l}20282 \\
12693\end{array}$ & $\begin{array}{l}9.033 \\
3.25\end{array}$ & $\begin{array}{l}0.20 \\
0.13\end{array}$ & $\begin{array}{l}21.63 \\
22.91\end{array}$ & $\begin{array}{r}8.14 \\
12.43\end{array}$ & $\begin{array}{l}.360 \\
0.90\end{array}$ & $\begin{array}{l}27.72 \\
16.44\end{array}$ & $\begin{array}{l}6.41 \\
6.46\end{array}$ & $\begin{array}{l}32.15 \\
37.48\end{array}$ \\
\hline Tepic & $\begin{array}{r}8475 \\
845\end{array}$ & 8.83 & 0.29 & $\begin{array}{l}22.97 \\
24.48\end{array}$ & $\begin{array}{l}7.0 .43 \\
11.07\end{array}$ & $\begin{array}{l}0.900 \\
0.25\end{array}$ & 18.43 & $\begin{array}{l}0.40 \\
6.75\end{array}$ & $\begin{array}{l}37.48 \\
29.90\end{array}$ \\
\hline Monterrey & 115703 & 1.48 & 0.73 & 37.53 & 8.93 & 0.77 & 19.40 & $\begin{array}{l}0.13 \\
10.06\end{array}$ & \\
\hline Oexaca & 17020 & 5.19 & 0.24 & 28.98 & 6.24 & 0.64 & 18.32 & 6.01 & 34.38 \\
\hline Puebla & 79765 & 1.47 & 0.14 & 37.34 & 5.98 & 0.81 & 18.22 & 8.92 & $\begin{array}{l}\begin{array}{l}34.50 \\
27.12\end{array} \\
-\end{array}$ \\
\hline Querétaro & 15429 & 7.37 & 0.02 & 30.35 & 3.39 & 0.47 & 19.76 & 5.52 & $\begin{array}{l}27.62 \\
27.62\end{array}$ \\
\hline San Luis Potosi & 42075 & 4.37 & 1.70 & 32.17 & 6.90 & 0.87 & 18.14 & 12.19 & 23.66 \\
\hline Culiacán & 13887 & 6.79 & 0.41 & 19.64 & 9.38 & 0.92 & 23.55 & 8.23 & 31.08 \\
\hline Mazatlån & 13666 & 7.24 & 0.33 & 24.02 & 6.52 & 0.69 & 20.34 & 16.23 & 24.63 \\
\hline Cd. Obregón & 10617 & 7.51 & 0.08 & 21.73 & 19,59 & 0.95 & 20.43 & 4.83 & 24.88 \\
\hline Hermosillo & 12973 & 5.94 & 0.71 & 23.49 & 11.26 & 0.88 & 20.14 & 6.11 & 31.47 \\
\hline Villahermosa & 10895 & 11.72 & 0.04 & 18.66 & 7.04 & 0.87 & 18.95 & 8.35 & 34.37 \\
\hline Cd. Victoria & 9291 & 10.93 & 0.10 & 13.92 & 10.74 & 0.83 & 21.26 & 9.61 & 32.61 \\
\hline Matamoros & 15347 & 12.38 & 0.32 & 19.46 & 6.11 & 0.52 & 28.18 & 6.99 & 25.54 \\
\hline Nuevo Laredo & 16829 & 6.62 & 0.99 & 16.79 & 9.14 & 0.80 & 20.09 & 14.29 & 32.08 \\
\hline Reynosa & 10787 & 9.29 & 3.14 & 16.77 & 9.98 & 0.63 & 22.81 & 7.73 & 29.65 \\
\hline Tampico & 42045 & 3.56 & 14.03 & 19.58 & 5.67 & 3.30 & 19.64 & 19.78 & 24.44 \\
\hline Jalapa & 17948 & 7.03 & 0.07 & 23.27 & 9.19 & 0.47 & 18.37 & $9 \cdot 39$ & 32.21 \\
\hline Orizaba & 24789 & 7.05 & 0.11 & 38.22 & 5.28 & 0.72 & 17.69 & 10.03 & 20.90 \\
\hline Veracruz & 34148 & 2.39 & 0.29 & 98.68 & 9.40 & 0.61 & 21.19 & 16.79 & 30.65 \\
\hline Mérida & 44058 & 5.04 & 0.06 & 28.21 & 9064 & 0.75 & 22.66 & 7.98 & 25.66 \\
\hline
\end{tabular}

Fuente: L. Unikel y F. Torres, "La población económicamente activa en Méśico y sus principales ciudades, 1940-1960", Democrafía y Economía, Vol. IV, Núm. 1, a Areas urbanas con 50000 habitantes y más en 1960 . c Los datos corresponden, por lo menos en uno de los tres años estudiados, a las áreas urbanas de estas ciudades -la ciudad central más la periferia de
contiguidad física--, excepto en once de ellas. Véase Unikel, "El proceso de urbanización...", loc. citt, cuadro 20 . 
Cuadro A 4

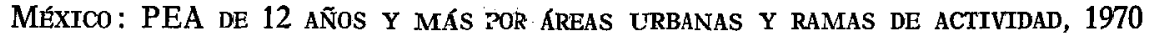
(Porcientos)

\begin{tabular}{|c|c|c|c|c|c|c|c|c|c|c|c|}
\hline \multirow[b]{2}{*}{ Manicipios } & \multirow[b]{2}{*}{$\begin{array}{l}\text { PEA } \\
\text { PotaI }\end{array}$} & \multicolumn{10}{|c|}{ Ramas de actividad } \\
\hline & & $\begin{array}{c}\text { Agrope } \\
\text { cuariala/ }\end{array}$ & $\begin{array}{l}\text { Petró } \\
100\end{array}$ & $\begin{array}{c}\text { Extrac } \\
\text { tiva }\end{array}$ & $\begin{array}{l}\text { Trans } \\
\text { forma } \\
\text { cion }\end{array}$ & $\underset{\text { cibn }}{\text { Construc }}$ & $\begin{array}{c}\text { Eleg } \\
\text { tricici } \\
\text { dad }\end{array}$ & $\begin{array}{c}\text { Comer } \\
\text { cio }\end{array}$ & $\begin{array}{l}\text { Comunioa } \\
\text { ciones } \\
\text { transpor } \\
\text { tes }\end{array}$ & $\begin{array}{l}\text { Servi } \\
\text { cios b }\end{array}$ & $\begin{array}{l}\text { Insuficien } \\
\text { temente es } \\
\text { peoificadas }\end{array}$ \\
\hline \multicolumn{12}{|l|}{ Total } \\
\hline Aguascalientes & 58190 & 20.46 & 0.17 & 0.21 & 19.91 & 5.67 & 0.35 & 12.83 & 9.86 & 21.97 & 8.57 \\
\hline Mexicali & 98738 & 33.24 & 0.12 & 0.32 & 15.39 & 4.38 & 0.55 & 12.63 & 2.75 & 23.87 & 6.80 \\
\hline Ti juana & 89013 & 9.19 & 0.18 & 0.31 & 21.27 & 7.17 & 0.53 & 16.93 & 3.29 & 32.24 & 8.89 \\
\hline Saltillo & 49475 & 15.42 & 0.13 & 1.20 & 25.86 & 7.34 & 0.49 & 11.64 & 5.21 & 25.33 & 7.38 \\
\hline Torrebn of & 112924 & 24.73 & 0.20 & 0.64 & 16.30 & 5.72 & 0.77 & 13.37 & 5.22 & 25.62 & 7.43 \\
\hline Cd. Juárez & 108078 & 8.65 & 0.18 & 0.37 & 17.78 & 8.19 & 0.39 & 17.72 & 4.19 & $34 \cdot 37$ & 8.16 \\
\hline Chihuahua of & $74 \quad 104$ & 11.99 & 0.23 & 1.32 & 17.85 & 7.73 & 0.67 & 14.70 & 6.24 & 32.27 & 7.00 \\
\hline Ca. de México & 2629979 & 2.32 & 0.55 & 0.28 & 31.03 & 5.90 & 0.65 & 13.69 & 4.22 & 36.92 & $4 \cdot 44$ \\
\hline Durango & 48687 & 26.57 & 0.10 & 1.01 & 13.98 & 6.07 & 0.45 & 11.51 & 5.16 & 27.08 & 8.07 \\
\hline Celaya & 38793 & 31.08 & 0.25 & 0.16 & 15.29 & 6.01 & 0.83 & 13.99 & 3.12 & 22.90 & 6.37 \\
\hline Irapuato & 44380 & 34.63 & 0.67 & 0.26 & 18.78 & 4.83 & 0.59 & 12.71 & 3.21 & 16.92 & 7.40 \\
\hline Leon & 110389 & 11.41 & 0.07 & 0.24 & 45.16 & $4 \cdot 39$ & 0.29 & 12.12 & 2.58 & 17.98 & 5.76 \\
\hline & 24490 & 5.69 & 0.25 & 7.66 & 19.85 & 6.12 & 0.87 & 14.72 & 3.71 & 35.29 & 5.84 \\
\hline $\begin{array}{l}\text { Guadala jara } \\
\text { Tol uca }\end{array}$ & 433890 & 6.22 & 0.11 & 0.35 & $\begin{array}{l}30.87 \\
23.52\end{array}$ & 8.06 & 0.48 & 15.17 & 4.68 & 27.70 & 6.36 \\
\hline $\begin{array}{l}\text { Toluca } \\
\text { Morelia }\end{array}$ & $\begin{array}{ll}65244 \\
51 & 167\end{array}$ & $\begin{array}{r}16.90 \\
20.82\end{array}$ & 0.11 & 0.25 & 23.52 & $\begin{array}{l}7.02 \\
7.38\end{array}$ & 0.62 & 12.11 & 3.70 & 27.97 & 7.80 \\
\hline $\begin{array}{l}\text { Morelia } \\
\text { Cuernavaca }\end{array}$ & $\begin{array}{lll}51 & 167 \\
48 & 447\end{array}$ & $\begin{array}{r}23.82 \\
0.36\end{array}$ & 0.13 & $\begin{array}{l}0.40 \\
0.39\end{array}$ & $\begin{array}{l}14.65 \\
-14\end{array}$ & 7.38 & 0.76 & 12.63 & 3.06 & 29.76 & $7 \cdot 41$ \\
\hline $\begin{array}{l}\text { Cuernavaca } \\
\text { Tepic }\end{array}$ & $\begin{array}{l}48447 \\
30444\end{array}$ & 9936 & $\begin{array}{l}0.14 \\
0.10\end{array}$ & $\begin{array}{l}0.39 \\
0.14\end{array}$ & 21.37 & $\begin{array}{l}9.23 \\
5.78\end{array}$ & 0.44 & 11.69 & 3.11 & 35.85 & 8.42 \\
\hline Monterrey & $\begin{array}{l}300444 \\
357822\end{array}$ & $\begin{array}{r}21.61 \\
3.05\end{array}$ & $\begin{array}{l}0.10 \\
0.18\end{array}$ & $\begin{array}{l}0.41 \\
0.46\end{array}$ & $\begin{array}{l}15.51 \\
36.32\end{array}$ & $\begin{array}{l}5.78 \\
7.89\end{array}$ & $\begin{array}{l}1.25 \\
0.41\end{array}$ & $\begin{array}{r}13.85 \\
13.44\end{array}$ & $\begin{array}{l}4.40 \\
4.57\end{array}$ & $\begin{array}{l}29.77 \\
28.93\end{array}$ & $\begin{array}{l}7.32 \\
4.75\end{array}$ \\
\hline Oaxaca & 42725 & 23.11 & 0.14 & 0.29 & 15.42 & 5.63 & 0.83 & 13.50 & 3.92 & 31.07 & 6.09 \\
\hline Pueb. & 154341 & 7.31 & 0.23 & 0.33 & 30.14 & 5.33 & 0.80 & 14.76 & 4.45 & 30.81 & 5.84 \\
\hline Querétaro & 44715 & 17.94 & 0.17 & 0.58 & 24.22 & 7.03 & 0.56 & 11.98 & 2.93 & 26.81 & 7.78 \\
\hline San Luis Potosf & 75277 & $11 \cdot 34$ & 0.22 & 1.45 & 22.81 & 7.27 & 0.53 & 13.52 & 7.82 & 28.12 & 6.92 \\
\hline Culiaosn & $\begin{array}{l}99061 \\
18\end{array}$ & 42.97 & 0.07 & 0.28 & 10.88 & 4.24 & 0.29 & 10.29 & 2.87 & 21.46 & 6.65 \\
\hline & $\begin{array}{l}48897 \\
46983\end{array}$ & 23.72 & 0.45 & 0.28 & 14.57 & $\begin{array}{r}5.49 \\
5.85\end{array}$ & 0.46 & 12.61 & 5.98 & 30.27 & 6.17 \\
\hline $\begin{array}{l}\text { cd. Cbregtin } \\
\text { Hermosillo }\end{array}$ & $\begin{array}{l}46983 \\
53552\end{array}$ & 29.34 & 0.21 & 0.38 & $11 \cdot 34$ & 5.85 & 0.42 & $15 \cdot 43$ & 3.45 & 27.71 & 5.87 \\
\hline $\begin{array}{l}\text { Hermosillo } \\
\text { Villahermosa }\end{array}$ & $\begin{array}{l}53552 \\
44235\end{array}$ & $\begin{array}{l}22.71 \\
33.88\end{array}$ & $\begin{array}{l}0.12 \\
1.44\end{array}$ & $\begin{array}{l}0.18 \\
0.17\end{array}$ & $\begin{array}{r}2.74 \\
9.31\end{array}$ & $\begin{array}{l}7.54 \\
5.53\end{array}$ & $\begin{array}{l}0.99 \\
0.63\end{array}$ & $\begin{array}{l}14.49 \\
12.48\end{array}$ & $\begin{array}{l}3.79 \\
2.76\end{array}$ & $\begin{array}{l}31.02 \\
26.63\end{array}$ & $\begin{array}{l}5.82 \\
7.17\end{array}$ \\
\hline Cd. Victoria & 24985 & 19.70 & 0.32 & 0.33 & 14.33 & 8.76 & 0.61 & 12.13 & 3.62 & 34.73 & 5.47 \\
\hline Matamoros & 39467 & 33.73 & 0.17 & 0.33 & 18.19 & 7.21 & 0.39 & 16.98 & 4.35 & 11.23 & 7.42 \\
\hline Naevo Laredo & 39463 & 11.14 & 0.16 & 0.30 & 19.72 & 6.80 & 0.37 & 14.47 & 4.76 & 34.66 & 7.62 \\
\hline Reynosa & 38032 & 16.10 & 14.45 & 0.24 & 10.77 & 7.78 & 0.38 & 14.07 & 3.40 & 25.93 & 6.88 \\
\hline Tampioos/ & $77 \quad 182$ & 5.26 & 13.37 & 0.37 & 13.41 & 8.43 & 0.63 & 15.25 & 5.25 & 32.62 & 5.41 \\
\hline Jalapa of & 37.407 & 11.02 & 0.34 & 0.21 & 12.55 & 7.67 & 1.38 & 14.21 & 5.67 & 38.94 & 8.01 \\
\hline Orizaba & 43037 & 8.71 & 0.50 & 0.38 & 33.73 & 5.53 & 0.90 & 14.47 & 4.91 & 25.12 & 5.75 \\
\hline Veraorue & $74 \quad 205$ & 6.31 & 1.33 & 0.15 & 20.53 & 5.82 & 0.80 & 14.98 & 6.16 & 36.44 & 7.48 \\
\hline Merida & 387 & 12.69 & 0.09 & 0.30 & 19.06 & 6.16 & 0.77 & 14.57 & 4.35 & 32.16 & 9.85 \\
\hline
\end{tabular}

Fuente: IX Censo General de Población 1970, Dirección General de Estadistica, SIC, 1971.

a Incluye silvicultura, caza y pesca.
b Incluye a la rama de actividad "gobierno".

c Estos casos abarcan a los municipios que contienen el área de las ciucades principales.
d Estas son las ciudades principales de los municipios de Cajeme y Centro, respectivamente. 


\section{Cuadro A 5}

MÉxICO: ÍNDICE DE DLIERENCIACIÓN ECONÓMICA (IED) SEGÚN LA PRIMERA FUNCIÓN PREDOMINANTE DE LAS CIUDADES, 1940-1960

\begin{tabular}{|c|c|c|c|c|c|}
\hline \multicolumn{2}{|l|}{1940} & \multicolumn{2}{|l|}{1950} & \multicolumn{2}{|l|}{1960} \\
\hline $\begin{array}{l}\text { Primera fancion } \\
\text { predominante }\end{array}$ & $I M D^{3}$ & $\begin{array}{l}\text { Primera fancion } \\
\text { predominante }\end{array}$ & $I E D^{a}$ & $\begin{array}{l}\text { Primera funcion } \\
\text { predominante }\end{array}$ & $I E D^{a /}$ \\
\hline $\begin{array}{c}\text { Wrtractiva } \\
\text { Pachaca } \\
\text { Pampico }\end{array}$ & $\begin{array}{l}0.084 \\
0.110 \\
0.059\end{array}$ & $\begin{array}{c}\text { Extractiva } \\
\text { Pachuca } \\
\text { Tampico }\end{array}$ & $\begin{array}{l}0.063 \\
0.075 \\
0.051\end{array}$ & $\begin{array}{c}\text { Extractiva } \\
\text { Pachuca } \\
\text { Indastrial }\end{array}$ & $\begin{array}{l}0.082 \\
0.082 \\
0.150\end{array}$ \\
\hline $\begin{array}{l}\text { Indostrial } \\
\text { Leon } \\
\text { Oaxaca } \\
\text { Querdiaro } \\
\text { Orizaba } \\
\text { Puebla } \\
\text { Irapdato } \\
\text { Oadalajara } \\
\text { Monterrey }\end{array}$ & $\begin{array}{l}0.167 \\
0.326 \\
0.178 \\
0.150 \\
0.149 \\
0.139 \\
0.136 \\
0.135 \\
0.120\end{array}$ & $\begin{array}{l}\text { Industrial } \\
\text { León } \\
\text { Orizaba } \\
\text { Querétaro } \\
\text { Monterrey } \\
\text { Gaadalajara } \\
\text { Constracoión } \\
\text { Cd. Juárez } \\
\text { Gd. Obregón }\end{array}$ & $\begin{array}{l}0.165 \\
0.300 \\
0.149 \\
0.133 \\
0.123 \\
0.118 \\
0.098 \\
0.102 \\
0.095\end{array}$ & $\begin{array}{l}\text { Lern } \\
\text { Orizaba } \\
\text { Puebla } \\
\text { Monterrey } \\
\text { Irapuato } \\
\text { Guadalajara } \\
\text { Aguascalientes } \\
\text { Tampico } \\
\text { Construccion }\end{array}$ & $\begin{array}{l}0.176 \\
0.276 \\
0.166 \\
0.147 \\
0.139 \\
0.130 \\
0.125 \\
0.110 \\
0.109 \\
0.109\end{array}$ \\
\hline $\begin{array}{l}\text { Congtrucoion } \\
\text { Cr. Cbregon } \\
\text { Comercial. }\end{array}$ & $\begin{array}{l}0.115 \\
0.115 \\
0.131\end{array}$ & $\begin{array}{l}\text { Comercial } \\
\text { Matamoros } \\
\text { Celaya }\end{array}$ & $\begin{array}{l}0.077 \\
0.111 \\
0.127 \\
0.123\end{array}$ & $\begin{array}{l}\text { Saltillo } \\
\text { Tepic } \\
\text { Chihuahua }\end{array}$ & $\begin{array}{l}0.149 \\
0.120 \\
0.111 \\
0.096\end{array}$ \\
\hline $\begin{array}{l}\text { Watameroa } \\
\text { Daremo } \\
\text { Celaya } \\
\text { Veriaja } \\
\text { CA. Juarez }\end{array}$ & $\begin{array}{l}0.147 \\
0.133 \\
0.131 \\
0.124 \\
0.121\end{array}$ & $\begin{array}{l}\text { Irapaato } \\
\text { Caliacán } \\
\text { Mérida } \\
\text { Torrén } \\
\text { Reynosa }\end{array}$ & $\begin{array}{l}0.118 \\
0.110 \\
0.108 \\
0.095 \\
0.095\end{array}$ & $\begin{array}{l}\text { Comercial } \\
\text { Matamoros } \\
\text { Nerida } \\
\text { Querétaro } \\
\text { Culiacan }\end{array}$ & $\begin{array}{l}0.116 \\
0.130 \\
0.129 \\
0.124 \\
0.115\end{array}$ \\
\hline $\begin{array}{l}\text { Mranaporte } \\
\text { Vidiahermosa } \\
\text { Varacouz } \\
\text { Mraevo Deredo }\end{array}$ & $\begin{array}{l}0.101 \\
0.123 \\
0.120 \\
0.116\end{array}$ & $\begin{array}{l}\text { Transporte } \\
\text { Paebla } \\
\text { San Lais Potosf } \\
\text { Aguascalientes }\end{array}$ & $\begin{array}{l}0.102 \\
0.143 \\
0.104 \\
0.101\end{array}$ & $\begin{array}{l}\text { Villahermosa } \\
\text { Cd. Obregón } \\
\text { Celaya } \\
\text { Reynosa }\end{array}$ & $\begin{array}{l}0.110 \\
0.109 \\
0.108 \\
0.107\end{array}$ \\
\hline $\begin{array}{l}\text { Mazatián } \\
\text { Saltillo } \\
\text { Agaraslientes } \\
\text { Torreön } \\
\text { San Laie Potosi }\end{array}$ & $\begin{array}{l}0.111 \\
0.103 \\
0.096 \\
0.086 \\
0.081\end{array}$ & $\begin{array}{l}\text { Naevo Laredo } \\
\text { Saltillo } \\
\text { Mazatián } \\
\text { Veracruz } \\
\text { Chihuahua }\end{array}$ & $\begin{array}{l}0.101 \\
0.101 \\
0.091 \\
0.091 \\
0.085\end{array}$ & $\begin{array}{l}\text { Transporte } \\
\text { San Lais Potosf } \\
\text { Torreón } \\
\text { Veracruz } \\
\text { Nazatlan }\end{array}$ & $\begin{array}{l}0.102 \\
0.109 \\
0.102 \\
0.101 \\
0.097\end{array}$ \\
\hline Chineahus & 0.075 & Servicios & 0.126 & Servicios & 0.131 \\
\hline $\begin{array}{l}\text { Servicios } \\
\text { Tijuana } \\
\text { Nexicali } \\
\text { Caennava } \\
\text { Tolaca } \\
\text { Coliact } \\
\text { Co. de Moxico } \\
\text { Morelia } \\
\text { Tepic } \\
\text { JElaca } \\
\text { Hermosillo } \\
\text { Reynosa } \\
\text { Co. Victoria }\end{array}$ & $\begin{array}{l}0.156 \\
0.222 \\
0.174 \\
0.172 \\
0.169 \\
0.159 \\
0.156 \\
0.140 \\
0.140 \\
0.137 \\
0.135 \\
0.135 \\
0.130\end{array}$ & $\begin{array}{l}\text { Oaraca } \\
\text { Ca. de Mexico } \\
\text { Villahermosa } \\
\text { mi juana } \\
\text { Cuernavaca } \\
\text { Toldoa } \\
\text { Mexicali } \\
\text { Norelia } \\
\text { Cd. Victoria } \\
\text { Jalapa } \\
\text { Tepic } \\
\text { Hermosillo } \\
\text { Daraneo }\end{array}$ & $\begin{array}{l}0.149 \\
0.138 \\
0.134 \\
0.133 \\
0.132 \\
0.131 \\
0.129 \\
0.129 \\
0.115 \\
0.115 \\
0.114 \\
0.111 \\
0.108\end{array}$ & $\begin{array}{l}\text { Oaxaca } \\
\text { Cd. de Mexico } \\
\text { Cuernavaca } \\
\text { Cd. Victoria } \\
\text { Tolnca } \\
\text { Morelia } \\
\text { Jalapa } \\
\text { Naevo Laredo } \\
\text { Ti juana } \\
\text { Cd. Jusrez } \\
\text { odexicali } \\
\text { Durango } \\
\text { Hermogillo }\end{array}$ & $\begin{array}{l}0.171 \\
0.144 \\
0.136 \\
0.135 \\
0.134 \\
0.133 \\
0.132 \\
0.131 \\
0.127 \\
0.119 \\
0.1119 \\
0.117 \\
0.106\end{array}$ \\
\hline
\end{tabular}

Cálculos elaborados según la fórmula que se presenta en el apéndice y con base en los datos de los cuadros A1, A2 y A3. 